\title{
NONLOCAL DISCRETE DIFFUSION EQUATIONS AND THE FRACTIONAL DISCRETE LAPLACIAN, REGULARITY AND APPLICATIONS
}

\author{
ÓSCAR CIAURRI, LUZ RONCAL, PABLO RAÚL STINGA, \\ JOSÉ L. TORREA, AND JUAN LUIS VARONA
}

\begin{abstract}
The analysis of nonlocal discrete equations driven by fractional powers of the discrete Laplacian on a mesh of size $h>0$

$$
\left(-\Delta_{h}\right)^{s} u=f
$$

for $u, f: \mathbb{Z}_{h} \rightarrow \mathbb{R}, 0<s<1$, is performed. The pointwise nonlocal formula for $\left(-\Delta_{h}\right)^{s} u$ and the nonlocal discrete mean value property for discrete $s$-harmonic functions are obtained. We observe that a characterization of $\left(-\Delta_{h}\right)^{s}$ as the Dirichlet-to-Neumann operator for a semidiscrete degenerate elliptic local extension problem is valid. Regularity properties and Schauder estimates in discrete Hölder spaces as well as existence and uniqueness of solutions to the nonlocal Dirichlet problem are shown. For the latter, the fractional discrete Sobolev embedding and the fractional discrete Poincaré inequality are proved, which are of independent interest. We introduce the negative power (fundamental solution)

$$
u=\left(-\Delta_{h}\right)^{-s} f,
$$

which can be seen as the Neumann-to-Dirichlet map for the semidiscrete extension problem. We then prove the discrete Hardy-Littlewood-Sobolev inequality for $\left(-\Delta_{h}\right)^{-s}$.

As applications, the convergence of our fractional discrete Laplacian to the (continuous) fractional Laplacian as $h \rightarrow 0$ in Hölder spaces is analyzed. Indeed, uniform estimates for the error of the approximation in terms of $h$ under minimal regularity assumptions are obtained. We finally prove that solutions to the Poisson problem for the fractional Laplacian
\end{abstract}

$$
(-\Delta)^{s} U=F,
$$

in $\mathbb{R}$, can be approximated by solutions to the Dirichlet problem for our fractional discrete Laplacian, with explicit uniform error estimates in terms of $h$.

\section{INTRODUCTION AND MAIN RESULTS}

The fractional Laplacian, understood as a positive power of the classical Laplacian, has been present for a long time in several areas of mathematics, like potential theory, harmonic analysis, fractional calculus, functional analysis and probability [10, 14, 16, 26]. However, although this operator appeared in some differential equations in physics [17, it was not until the past decade when it became a very popular object in the field of partial differential

2010 Mathematics Subject Classification. Primary: 35R11, 49M25. Secondary: 35K05, 65N15.

Key words and phrases. Nonlocal discrete diffusion equations, fractional discrete Laplacian, regularity and extension problem, Sobolev and Poincaré inequalities, error of approximation, semidiscrete heat equation.

Research partially supported by grants MTM2015-66157-C2-1-P and MTM2015-65888-C04-4-P, MINECO/FEDER, UE, from Government of Spain. L.R. was also supported by the Basque Government through the BERC 2018-2021 program, by Spanish Ministry of Economy and Competitiveness MINECO through BCAM Severo Ochoa excellence accreditation SEV-2013-0323 and through project MTM2017-82160C2-1-P funded by (AEI/FEDER, UE) and acronym "HAQMEC", and by a 2017 Leonardo grant for Researchers and Cultural Creators, BBVA Foundation. The Foundation accepts no responsibility for the opinions, statements and contents included in the project and/or the results thereof, which are entirely the responsibility of the authors. 
equations. Indeed, nonlocal diffusion equations involving fractional Laplacians have been one of the most studied research topics in the present century. The fractional Laplacian on $\mathbb{R}^{n}$ is defined, for $0<s<1$ and good enough functions $U$, as

$$
(-\Delta)^{s} U(x)=c_{n, s} \mathrm{P} . \mathrm{V} \cdot \int_{\mathbb{R}^{n}} \frac{U(x)-U(y)}{|x-y|^{n+2 s}} d y,
$$

for $x \in \mathbb{R}^{n}$, where $c_{n, s}>0$ is an explicit constant, see [14, 29]. We could say that the triggers that produced the outbreak in the field were the papers by L. Caffarelli and L. Silvestre [4] and L. Silvestre [25]. Since the appearance of those works there has been a substantial revision of a big amount of problems in differential equations where the Laplacian is replaced by the fractional Laplacian or more general integro-differential operators, see for example [3, 5, 9, 21, 22, 23, 24, 25, 28, 29, and references therein for models and techniques. On the other hand, there is the basic question of approximating the continuous problems by discrete ones. The large literature includes numerical approximations of different sorts, see for example [1, 2, 8, 12, 13, 18, and references therein. The main difficulties to overcome in numerical approaches are the nonlocality and singularity of the operator (1.1). In any case, it is expected for discrete jump models to approximate continuous jump models in a good way as the size of the mesh goes to zero [17, a question that we also address here.

One of our aims in this paper is to present a quite complete study of nonlocal discrete diffusion equations involving the fractional powers of the discrete Laplacian

$$
\left(-\Delta_{h}\right)^{s} u
$$

and show how they can be used to approximate solutions to the Poisson problem for the fractional Laplacian

$$
(-\Delta)^{s} U=F, \quad \text { in } \mathbb{R} .
$$

We describe next our main results.

Along the paper we consider a mesh of fixed size $h>0$ on $\mathbb{R}$ given by $\mathbb{Z}_{h}=\{h j: j \in \mathbb{Z}\}$. For a function $u: \mathbb{Z}_{h} \rightarrow \mathbb{R}$ we use the notation $u_{j}=u(h j)$ to denote the value of $u$ at the mesh point $h j \in \mathbb{Z}_{h}$. The discrete Laplacian $\Delta_{h}$ on $\mathbb{Z}_{h}$ is then given by

$$
-\Delta_{h} u_{j}=-\frac{1}{h^{2}}\left(u_{j+1}-2 u_{j}+u_{j-1}\right) \text {. }
$$

For $0<s<1$, we define the fractional powers of the discrete Laplacian $\left(-\Delta_{h}\right)^{s} u$ on $\mathbb{Z}_{h}$ with the semigroup method (see [28, 29]) as

$$
\left(-\Delta_{h}\right)^{s} u_{j}=\frac{1}{\Gamma(-s)} \int_{0}^{\infty}\left(e^{t \Delta_{h}} u_{j}-u_{j}\right) \frac{d t}{t^{1+s}}
$$

Here $w_{j}(t)=e^{t \Delta_{h}} u_{j}$ is the solution to the semidiscrete heat equation

$$
\begin{cases}\partial_{t} w_{j}=\Delta_{h} w_{j}, & \text { in } \mathbb{Z}_{h} \times(0, \infty), \\ w_{j}(0)=u_{j}, & \text { on } \mathbb{Z}_{h},\end{cases}
$$

(see Section 22) and $\Gamma$ denotes the Gamma function. As we will see, our technique provides a formula that gives the exact solution of this equation and that is central along the paper. This can be applied for fixed $h>0$, but obviously not for non-uniform meshes, for instance.

Theorem 1.1 (Pointwise nonlocal formula and limits). For $0 \leq s \leq 1$, we let

$$
\ell_{ \pm s}:=\left\{u: \mathbb{Z}_{h} \rightarrow \mathbb{R}:\|u\|_{\ell_{ \pm s}}:=\sum_{m \in \mathbb{Z}} \frac{\left|u_{m}\right|}{(1+|m|)^{1 \pm 2 s}}<\infty\right\}
$$


(a) If $0<s<1$ and $u \in \ell_{s}$ then

$$
\left(-\Delta_{h}\right)^{s} u_{j}=\sum_{m \in \mathbb{Z}, m \neq j}\left(u_{j}-u_{m}\right) K_{s}^{h}(j-m),
$$

where the discrete kernel $K_{s}^{h}$ is given by

$$
K_{s}^{h}(m)=\frac{4^{s} \Gamma(1 / 2+s)}{\sqrt{\pi}|\Gamma(-s)|} \cdot \frac{\Gamma(|m|-s)}{h^{2 s} \Gamma(|m|+1+s)},
$$

for any $m \in \mathbb{Z} \backslash\{0\}$, and $K_{s}^{h}(0)=0$.

(b) For $0<s<1$ there exist constants $0<c_{s} \leq C_{s}$ such that, for any $m \in \mathbb{Z} \backslash\{0\}$,

$$
\frac{c_{s}}{h^{2 s}|m|^{1+2 s}} \leq K_{s}^{h}(m) \leq \frac{C_{s}}{h^{2 s}|m|^{1+2 s}} .
$$

(c) If $u \in \ell_{0}$ then $\lim _{s \rightarrow 0^{+}}\left(-\Delta_{h}\right)^{s} u_{j}=u_{j}$.

(d) If $u$ is bounded then $\lim _{s \rightarrow 1^{-}}\left(-\Delta_{h}\right)^{s} u_{j}=-\Delta_{h} u_{j}$.

The expression in (1.6) and the estimate in (1.8) show that the fractional discrete Laplacian is a nonlocal operator on $\mathbb{Z}_{h}$ of order $2 s$ (we precise this in Theorems 1.5 and 1.6). Notice also that our definition 1.3 is neither a direct discretization of the pointwise formula for the fractional Laplacian (1.1), nor a "discrete analogue", but the $s$-fractional power of the discrete Laplacian. In this regard, we warn the reader that the notation $\left(-\Delta_{h}\right)^{\alpha / 2}, 0<\alpha<2$, used in [12] does not refer to the fractional power of the discrete Laplacian (1.6), but to a specific way of discretizing the pointwise formula in (1.1). The constant

$$
A_{s}:=\frac{4^{s} \Gamma(1 / 2+s)}{\sqrt{\pi}|\Gamma(-s)|}>0
$$

which appears in the kernel $K_{s}^{h}(m)$, see (1.7), is exactly the same constant $c_{n, s}>0$ in the formula for the fractional Laplacian (1.1) when $n=1$.

Remark 1.2 (Mean value formula and probabilistic interpretation). Let $u$ be a discrete harmonic function on $\mathbb{Z}_{h}$, that is, $-\Delta_{h} u=0$. This is equivalent as saying that $u$ satisfies the discrete mean value property:

$$
u_{j}=\frac{1}{2} u_{j+1}+\frac{1}{2} u_{j-1} .
$$

This identity shows that a discrete harmonic function describes the random movement of a particle that jumps either to the adjacent left point or to the adjacent right point with probability $1 / 2$. Suppose now that $u$ is a fractional discrete harmonic function, that is, $\left(-\Delta_{h}\right)^{s} u_{j}=0$. Then from 1.6 we have the following nonlocal mean value property:

$$
u_{j}=\frac{1}{\sum_{s}^{h}} \sum_{m \in \mathbb{Z}, m \neq j} u_{m} K_{s}^{h}(j-m)=: \sum_{m \in \mathbb{Z}} u_{m} P_{s}(j-m),
$$

where $\Sigma_{s}^{h}:=\sum_{m \in \mathbb{Z}} K_{s}^{h}(m)=A_{s} h^{-2 s} / s=\frac{4^{s} \Gamma(1 / 2+s)}{h^{2 s} \sqrt{\pi} \Gamma(1+s)}$, so that $P_{s}(m)$ is a probability density on $\mathbb{Z}$ with $P_{s}(0)=0$ which is independent of $h>0$. In a parallel way we understand this last identity by saying that a fractional discrete harmonic function describes a particle that is allowed to randomly jump to any point on $\mathbb{Z}_{h}$ (not only to the adjacent ones) and that the probability to jump from the point $h j$ to the point $h m$ is $P_{s}(j-m)$. By (1.8) this probability is proportional to $|j-m|^{-(1+2 s)}$. As $s \rightarrow 1^{-}$the probability to jump from $j$ to a non adjacent point tends to zero, while the probability to jump to an adjacent point tends 
to one, recovering in this way the previous situation. As $s \rightarrow 0^{+}$, the probability to jump to any point tends to zero, so there are no jumps.

The solution to the fractional discrete Poisson problem $\left(-\Delta_{h}\right)^{s} u=f$ in $\mathbb{Z}_{h}$ is realized by the negative powers of the discrete Laplacian, which are also called the fractional discrete integrals. They are defined, for $s>0$ and a function $f: \mathbb{Z}_{h} \rightarrow \mathbb{R}$, as

$$
\left(-\Delta_{h}\right)^{-s} f_{j}=\frac{1}{\Gamma(s)} \int_{0}^{\infty} e^{t \Delta_{h}} f_{j} \frac{d t}{t^{1-s}} \text {. }
$$

The kernel of $\left(-\Delta_{h}\right)^{-s}$ is the fundamental solution of $\left(-\Delta_{h}\right)^{s}$.

Theorem 1.3 (Fundamental solution and Hardy-Littlewood-Sobolev inequality). Let us fix $0<s<1 / 2$ and let $f \in \ell_{-s}$ (see (1.5)).

(a) We have the pointwise formula

$$
\left(-\Delta_{h}\right)^{-s} f_{j}=\sum_{m \in \mathbb{Z}} K_{-s}^{h}(j-m) f_{m}
$$

where, for $m \in \mathbb{Z}$, the discrete kernel $K_{-s}^{h}(m)$ is given by

$$
K_{-s}^{h}(m)=\frac{4^{-s} \Gamma(1 / 2-s)}{\sqrt{\pi} \Gamma(s)} \cdot \frac{\Gamma(|m|+s)}{h^{-2 s} \Gamma(|m|+1-s)} .
$$

(b) There exist positive constants $c_{s}, C_{s}$ and $d_{s} \leq D_{s}$ such that, for $m \in \mathbb{Z} \backslash\{0\}$,

$$
\frac{d_{s}}{h^{-2 s}|m|^{1-2 s}} \leq K_{-s}^{h}(m) \leq \frac{D_{s}}{h^{-2 s}|m|^{1-2 s}},
$$

and

$$
\left|K_{-s}^{h}(m)-\frac{c_{s}}{h^{-2 s}|m|^{1-2 s}}\right| \leq \frac{C_{s}}{h^{-2 s}|m|^{2-2 s}} .
$$

(c) Let $1<p<q<\infty$ such that $1 / q \leq 1 / p-2 s$. There exists a constant $C>0$, depending only on $p, q$ and $s$, such that if $f \in \ell_{h}^{p}$ (see (3.1) ) then $\left(-\Delta_{h}\right)^{-s} f \in \ell_{h}^{q}$ and

$$
\left\|\left(-\Delta_{h}\right)^{-s} f\right\|_{\ell_{h}^{q}} \leq \frac{C}{h^{1 / p-2 s-1 / q}}\|f\|_{\ell_{h}^{p}}
$$

It is worth comparing formula $(1.12$ for the kernel of the fractional discrete integral $K_{-s}^{h}(m)$ with the one for the kernel of the fractional discrete Laplacian $K_{s}^{h}(m)$ in (1.7). We also point out that the factor of $h$ disappears from the right hand side of 1.15$)$ when we reach the critical exponent $q=p /(1-2 s p)$. As before, $(1.11)$ is the $(-s)$-power of the discrete Laplacian, not a "discrete analogue" as that of [27]. Observe that the constant

$$
A_{-s}:=\frac{4^{-s} \Gamma(1 / 2-s)}{\sqrt{\pi} \Gamma(s)}>0,
$$

appearing in the kernel $K_{-s}^{h}$, see 1.12 , is exactly the same normalizing constant for the fractional integral $(-\Delta)^{-s}$ in dimension one in which $0<s<n / 2, n=1$, see [26] and Theorem 9.9 .

Remark 1.4 (Extension problem for $\left(-\Delta_{h}\right)^{s}$ and $\left(-\Delta_{h}\right)^{-s}$ ). The fractional powers of the discrete Laplacian which, as we have seen in Theorems 1.1] and 1.3, are nonlocal discrete operators, can be regarded as boundary values (Dirichlet or Neumann) of the solution to a local semidiscrete elliptic extension problem. This observation is just an application of the general extension problem of [28, 29], see also [9]. Thus, the positive powers can be seen as 
Dirichlet-to-Neumann maps, while the negative ones as Neumann-to-Dirichlet maps. Indeed, consider the semidiscrete degenerate elliptic operator

$$
L_{a, h}=\Delta_{h}+\frac{a}{y} \partial_{y}+\partial_{y y}
$$

where $a=1-2 s$ and $0<s<1$. This operator acts on semidiscrete functions $w=w_{j}(y)=$ $w(h j, y): \mathbb{Z}_{h} \times(0, \infty) \rightarrow \mathbb{R}$. Given $u \in \operatorname{Dom}\left(\left(-\Delta_{h}\right)^{s}\right)$, the semidiscrete function $w$ defined as

$$
w_{j}(y)=\frac{y^{2 s}}{4^{s} \Gamma(s)} \int_{0}^{\infty} e^{-y^{2} /(4 t)} e^{t \Delta_{h}} u_{j} \frac{d t}{t^{1+s}}
$$

is the unique solution (weakly vanishing as $y \rightarrow \infty$ ) to the Dirichlet problem

$$
\begin{cases}L_{a, h} w=0, & \text { in } \mathbb{Z}_{h} \times(0, \infty), \\ w_{j}(0)=u_{j}, & \text { on } \mathbb{Z}_{h}\end{cases}
$$

Moreover,

$$
-\lim _{y \rightarrow 0^{+}} y^{a} \partial_{y} w_{j}(y)=-2 s \lim _{y \rightarrow 0^{+}} \frac{w_{j}(y)-w_{j}(0)}{y^{2 s}}=\frac{\Gamma(1-s)}{4^{s-1 / 2} \Gamma(s)}\left(-\Delta_{h}\right)^{s} u_{j} .
$$

Analogously, given $f \in \operatorname{Dom}\left(\left(-\Delta_{h}\right)^{-s}\right)$, the semidiscrete function $v$ defined as

$$
v_{j}(y)=\frac{1}{\Gamma(s)} \int_{0}^{\infty} e^{-y^{2} /(4 t)} e^{t \Delta_{h}} f_{j} \frac{d t}{t^{1-s}}
$$

is the unique solution (weakly vanishing as $y \rightarrow \infty$ ) to the Neumann problem

$$
\begin{cases}L_{a, h} v=0 & \text { in } \mathbb{Z}_{h} \times(0, \infty), \\ -\left.y^{a} \partial_{y} v_{j}(y)\right|_{y=0^{+}}=f_{j}, & \text { on } \mathbb{Z}_{h} .\end{cases}
$$

Moreover,

$$
\lim _{y \rightarrow 0^{+}} v_{j}(y)=\frac{4^{s-1 / 2} \Gamma(s)}{\Gamma(1-s)}\left(-\Delta_{h}\right)^{-s} f_{j}
$$

It is obvious that if we have $\left(-\Delta_{h}\right)^{s} u=\frac{4^{s-1 / 2} \Gamma(s)}{\Gamma(1-s)} f$ then $w=v$.

We next go back to the fractional discrete Laplacian and show that it behaves as a fractional discrete derivative of order $2 s$ in discrete Hölder spaces. This will be obtained by exploiting (1.6). The following estimates are parallel to the corresponding ones for the fractional Laplacian (see [25]). For the definition of discrete Hölder spaces $C_{h}^{k, \alpha}$ see Definition 4.2 .

Theorem 1.5 (Fractional discrete Laplacian in discrete Hölder spaces). Let $k \geq 0,0<\alpha \leq$ $1,0<s<1$ and $u \in \ell_{s}$ (see 1.5 ).

(i) If $u \in C_{h}^{k, \alpha}$ and $2 s<\alpha$ then $\left(-\Delta_{h}\right)^{s} u \in C_{h}^{k, \alpha-2 s}$ and

$$
\left[\left(-\Delta_{h}\right)^{s} u\right]_{C_{h}^{k, \alpha-2 s}} \leq C[u]_{C_{h}^{k, \alpha}} .
$$

(ii) If $u \in C_{h}^{k+1, \alpha}$ and $2 s>\alpha$ then $\left(-\Delta_{h}\right)^{s} u \in C_{h}^{k, \alpha-2 s+1}$ and

$$
\left[\left(-\Delta_{h}\right)^{s} u\right]_{C_{h}^{k, \alpha-2 s+1}} \leq C[u]_{C_{h}^{k+1, \alpha}}
$$

The constants $C>0$ appearing above are independent of $h>0$ and $u$.

The following result, which complements Theorem 1.5, contains the discrete Schauder estimates for the fractional discrete Laplacian.

Theorem 1.6 (Discrete Schauder estimates). Let $k \geq 0,0<\alpha \leq 1,0<s<1 / 2$ and $f \in \ell_{-s}($ see 1.5$)$. 
(i) If $f \in C_{h}^{k, \alpha}$ and $2 s+\alpha<1$ then $\left(-\Delta_{h}\right)^{-s} f \in C_{h}^{k, \alpha+2 s}$ and

$$
\left[\left(-\Delta_{h}\right)^{-s} f\right]_{C_{h}^{k, \alpha+2 s}} \leq C[f]_{C_{h}^{k, \alpha}} .
$$

(ii) If $f \in C_{h}^{k, \alpha}$ and $2 s+\alpha>1$ then $\left(-\Delta_{h}\right)^{-s} f \in C_{h}^{k+1, \alpha+2 s-1}$ and

$$
\left[\left(-\Delta_{h}\right)^{-s} f\right]_{C_{h}^{k+1, \alpha+2 s-1}} \leq C[f]_{C_{h}^{k, \alpha}} .
$$

(iii) If $f \in \ell_{h}^{\infty}$, see 3.2 , then $\left(-\Delta_{h}\right)^{-s} f \in C_{h}^{0,2 s}$ and

$$
\left[\left(-\Delta_{h}\right)^{-s} f\right]_{C_{h}^{0,2 s}} \leq C\|f\|_{\ell_{h}^{\infty}}
$$

The constants $C>0$ appearing above are independent of $h>0$ and $f$.

Next we present what might be considered the most interesting results of this paper. We show how the fractional discrete Laplacian approximates the fractional Laplacian as $h \rightarrow 0$ in the strongest possible sense, that is, in the uniform norm. We need some notation. Given a function $U=U(x): \mathbb{R} \rightarrow \mathbb{R}$, we define its restriction $r_{h} U: \mathbb{Z}_{h} \rightarrow \mathbb{R}$ to the mesh $\mathbb{Z}_{h}$ to be the discrete function (or sequence) $\left(r_{h} U\right)_{j}:=U(h j)$, for $h j \in \mathbb{Z}_{h}$. The first approximation result considers uniform estimates for differences of the type

$$
\left\|\left(-\Delta_{h}\right)^{s}\left(r_{h} U\right)-r_{h}\left((-\Delta)^{s} U\right)\right\|_{\ell_{h}^{\infty}}
$$

in terms of the size $h$ of the mesh. The estimates will certainly depend on the regularity of $U$, which we take to be in a Hölder space $C^{k, \alpha}$ (see Definition 4.1). The notation $D_{+} u$ refers to the discrete derivative of $u: \mathbb{Z}_{h} \rightarrow \mathbb{R}$, see 4.1.

Theorem 1.7 (Uniform comparison with fractional Laplacian). Let $0<\alpha \leq 1,0<s<1$.

(i) If $U \in C^{0, \alpha}$ and $2 s<\alpha$ then

$$
\left\|\left(-\Delta_{h}\right)^{s}\left(r_{h} U\right)-r_{h}\left((-\Delta)^{s} U\right)\right\|_{\ell_{h}^{\infty}} \leq C[U]_{C^{0, \alpha}} h^{\alpha-2 s} .
$$

(ii) If $U \in C^{1, \alpha}$ and $2 s<\alpha$ then

$$
\left\|D_{+}\left(-\Delta_{h}\right)^{s}\left(r_{h} U\right)-r_{h}\left(\frac{d}{d x}(-\Delta)^{s} U\right)\right\|_{\ell_{h}^{\infty}} \leq C[U]_{C^{1, \alpha}} h^{\alpha-2 s} .
$$

(iii) If $U \in C^{1, \alpha}$ and $\alpha<2 s<1+\alpha$ then

$$
\left\|\left(-\Delta_{h}\right)^{s}\left(r_{h} U\right)-r_{h}\left((-\Delta)^{s} U\right)\right\|_{\ell_{h}^{\infty}} \leq C[U]_{C^{1, \alpha}} h^{\alpha-2 s+1} .
$$

(iv) If $U \in C^{k, \alpha}$ and $k+\alpha-2 s$ is not an integer then

$$
\left\|D_{+}^{l}\left(-\Delta_{h}\right)^{s}\left(r_{h} U\right)-r_{h}\left(\frac{d^{l}}{d x^{l}}(-\Delta)^{s} U\right)\right\|_{\ell_{h}^{\infty}} \leq C[U]_{C^{k, \alpha}} h^{\alpha-2 s+k-l},
$$

where $l$ is the integer part of $k+\alpha-2 s$.

The constants $C>0$ appearing above are independent of $h$ and $U$.

Although the proof of Theorem 1.7 is not trivial, one could say in a very naïve way that such a result is in some sense announced by Theorem 1.5. Indeed, the fractional discrete Laplacian maps $C_{h}^{\beta}$ into $C_{h}^{\beta-2 s}$. The continuous version of this property is also true for the fractional Laplacian, so the restriction of $(-\Delta)^{s} U$ to the mesh $\mathbb{Z}_{h}$ is in $C_{h}^{\beta-2 s}$ whenever $U \in C^{\beta}$. We also point out that $D_{+}$in Theorem 1.7 can be replaced by $D_{-}$, see (4.1).

The second approximation statement is the convergence of discrete solutions to continuous ones: the solution to the Poisson problem for the fractional Laplacian $(1.2)$ can be approximated by using the solution to the Dirichlet problem for the fractional discrete Laplacian (1.17). For $R>0$, we set $B_{R}^{h}=\left\{h j \in \mathbb{Z}_{h}:|h j|<R\right\}$ and $B_{R}=(-R, R) \subset \mathbb{R}$. 
Theorem 1.8 (Convergence of discrete solutions to continuous ones). Let $0<\alpha, s<1$ such that $\alpha+2 s<1$. Let $F \in C^{0, \alpha}$ with compact support contained in an interval $B_{R_{0}}$, for some $R_{0}>0$. Let $U \in C^{0, \alpha+2 s}$ be the unique solution to the Poisson problem (1.2) vanishing at infinity (see Theorem 9.9 where this function $U$ is explicitly constructed). Fix $h>0$ and let $f=r_{h} F$, the restriction of $F$ to $\mathbb{Z}_{h}$. Let $u: \mathbb{Z}_{h} \rightarrow \mathbb{R}$ be the unique solution (provided by Theorem 6.1) to the discrete Dirichlet problem

$$
\begin{cases}\left(-\Delta_{h}\right)^{s} u=f, & \text { in } B_{R}^{h}, \\ u=0, & \text { in } \mathbb{Z}_{h} \backslash B_{R}^{h},\end{cases}
$$

where $R>\max \left\{2 R_{0}, h^{-\alpha}\right\}$. Then there is a constant $C>0$, depending on $s, \alpha$ and $R_{0}$, but not on $R$ or $h$, such that

$$
\left\|u-r_{h} U\right\|_{\ell_{h}^{\infty}\left(B_{R}^{h}\right)} \leq C\|F\|_{C^{0, \alpha}} R^{2 s} h^{\alpha} .
$$

As far as the authors are aware, Theorem 1.8 is the first result where error estimates in the $L^{\infty}$-norm for approximations of solutions to the Poisson problem for the fractional Laplacian by a nonlocal discrete problem are proved. We stress that the Poisson problem $(-\Delta)^{s} U=F$ in Hölder spaces is non variational and $U$ and $u$ are classical solutions. In the discrete problem (1.17) a Dirichlet boundary condition means to prescribe the values of $u$ outside $B_{R}^{h}$ because of the nonlocality of the fractional discrete Laplacian, see 1.6 .

On the part of the domain that is left out of the estimate (1.18), that is, outside of $B_{R}^{h}$, we are approximating $U$ by the zero function so, in particular,

$$
\left\|u-r_{h} U\right\|_{\ell_{h}^{\infty}\left(\mathbb{Z}_{h} \backslash B_{R}^{h}\right)}=\left\|r_{h} U\right\|_{\ell_{h}^{\infty}\left(\mathbb{Z}_{h} \backslash B_{R}^{h}\right)} \leq C \frac{\|F\|_{L^{\infty}}}{R^{1-2 s}},
$$

where $C>0$ depends only on $s$, see (8.1) and also Theorem 9.9. This estimate is sharp. Indeed, suppose that

$$
\chi_{[-1,1]}(x) \leq F(x) \leq \chi_{[-2,2]}(x), \quad \text { for every } x \in \mathbb{R} .
$$

Then, for any $x>4$, in the notation of Theorem 9.9 ,

$$
U(x)=A_{-s} \int_{\mathbb{R}} \frac{F(y)}{|x-y|^{1-2 s}} d y \geq A_{-s} \int_{0}^{1} \frac{1}{(x-y)^{1-2 s}} d y \geq \frac{C_{s}}{x^{1-2 s}} .
$$

Another important fact we state in Theorem 1.8 is the unique solvability of the discrete Dirichlet problem (1.17). We show this in Section 6, see Theorem 6.1. On the way we need to prove the fractional discrete Sobolev embedding

$$
\|u\|_{\ell_{h}^{2 /(1-2 s)}} \leq C_{s}\left\|\left(-\Delta_{h}\right)^{s / 2} u\right\|_{\ell_{h}^{2}}
$$

(in which $s<1 / 2$ ) and obtain as a consequence, see Theorem 6.4, the fractional discrete Poincaré inequality

$$
\|u\|_{\ell_{h}^{2}} \leq C_{s} h^{s}\left(\# h_{h} \operatorname{supp}(u)\right)^{s}\left\|\left(-\Delta_{h}\right)^{s / 2} u\right\|_{\ell_{h}^{2}},
$$

where $\#_{h} E$ denotes the number of points in the set $E$. The proof of the latter inequality is postponed until Subsection 9.3 .

Theorem 1.8 will then be a consequence of Theorem 1.5 $(i)$ and the nonlocal discrete maximum principle we prove in Section 7, see Theorem 7.1. The presence of the factor $R^{2 s}$ in 1.18 is actually natural in view of such a maximum principle.

We also claim in Theorem 1.8 the existence of a unique, explicitly computed, classical solution $U$ to the Poisson problem (1.2). Though we believe this statement belongs to the folklore, we will present a self contained proof showing that such solution is indeed $U(x)=(-\Delta)^{-s} F(x)$, see Theorem 9.9. Note that $(-\Delta)^{-s}$ defines a tempered distribution 
on $\mathbb{R}$ if and only if $s<1 / 2$, see [25]. In addition, both the minimal regularity hypothesis $0<\alpha+2 s<1$ and the explicit formula for $U$ in Theorem 9.9 in terms of $(-\Delta)^{-s}$, as well as the assumption $2 s<\alpha$ in Theorem 1.7 $(i)$, that are used in the proof of Theorem 1.8, imply $0<s<1 / 2$. The same range of $s$ is considered in [23] and [25]. On the other hand $U$ has the minimal regularity. Hence no extra smoothness other than the correct one is assumed.

In an extremely naïve way, and parallel to Theorem 1.7. one could think that estimate (1.18) should be suggested by the value of the difference

$$
\left\|\left(-\Delta_{h}\right)^{-s}\left(r_{h} F\right)-r_{h}\left((-\Delta)^{-s} F\right)\right\|_{\ell_{h}^{\infty}} .
$$

However, in this case the situation is completely different. This is due to the absence of information about $\left(-\Delta_{h}\right)^{s} u$ in $\mathbb{Z}_{h} \backslash B_{R}^{h}$. Even more, in our Theorem 1.8 the presence of $R$ is essential as we showed a few lines above. Of course, different types of discrete problems could be chosen to approximate the Poisson problem (1.2). Our results could also raise the question of the approximation of the solution of the Dirichlet problem

$$
(-\Delta)^{s} V=G \text { in } B_{R}, \quad V=0 \text { in } \mathbb{R} \backslash B_{R},
$$

but we observe that, in this case, a preliminary discussion about the appropriate definition of $(-\Delta)^{s}$ that is being used (regional, restriction of the global, etc) should be given.

It is important to notice that the optimal Hölder regularity of the solution $u$ to the discrete Dirichlet problem (1.17) is not known. We conjecture that $u \in C_{h}^{0, s}$. In any case, $u$, being a sequence with only finitely many nonzero terms, is a classical solution and our error estimate (1.18) is explicit in terms of $h$ and $R$. Observe that, as $h$ tends to zero, the solution $u$ in (1.17) must be found in a larger domain $B_{R}^{h}$.

One of the main strategies used to obtain our results is the method of semigroups. Since the semidiscrete heat semigroup is given in terms of modified Bessel functions, see Section 2 , we will exhaustively use some properties and facts about these functions that we collect in Subsection 9.2 .

Some of our results can be easily extended to higher dimensions (for example, the extension problem in Remark 1.4) and we leave this task to the interested reader. In fact, it is possible to define the multi-dimensional discrete Laplacian and explicitly write down the solution to the corresponding semidiscrete heat equation (which is a key tool along our paper) as a discrete convolution with a heat kernel given as a product of Bessel functions of different integer orders. In this paper we present several fine results involving precise estimates of Bessel and Gamma functions that appear to be quite non trivial to mimic in higher dimensions. The semigroup method we use is obviously independent of the dimension, so semigroup formulas for fractional powers of multidimensional discrete Laplacians can be written down. However, closed pointwise formulas as explicit as the ones we discovered in Theorems 1.1 and 1.3 , that are crucial along the paper, will not be available anymore. Thus a different method must be found to prove our results in higher dimensions and we pose this together with the Hölder regularity of the solution $u$ to (1.17) as open problems. In any case, not only our main results are certainly novel, but also our techniques, which involve the manipulation of the semidiscrete heat equation. We mention related questions raised in [16, 31]. Those works deal with discrete Laplacians only at the level of $L^{2}$-spaces. Instead, we work with Hölder spaces, presenting estimates in the uniform norm (in this regard, our paper does not deal with variational problems and techniques) with explicit dependence on $h$. As a matter of fact, all our estimates recover the continuous ones as $h \rightarrow 0^{+}$.

The structure of the paper is as follows. Section 2 is devoted to the proof of Theorem 1.1 . In Section 3 we prove Theorem 1.3. The proofs of Theorems 1.5 and 1.7 are presented in Section 4. Section 5 contains the proof of Theorem 1.6. The Dirichlet problem for the fractional 
discrete Laplacian is analyzed in Section 6 . Section 7 contains the discrete maximum principle. The proof of Theorem 1.8 is done in Section 8. Some technical lemmas, the properties of Bessel functions, the proofs of the fractional discrete Sobolev and Poincaré inequalities and the analysis of the Poisson problem (1.2) are all collected in Section 9. By $C_{s}, c_{s}, D_{s}, d_{s}$ we mean positive constants depending on $s$ that may change in each occurrence, while by $C$ we will denote a constant independent of the significant variables. The notation $B^{h}$ refers to generic discrete finite interval contained in $\mathbb{Z}_{h}$.

\section{Proof of Theorem 1.1}

Given $u: \mathbb{Z}_{h} \rightarrow \mathbb{R}$, the solution to the semidiscrete heat equation (1.4) can be written as

$$
e^{t \Delta_{h}} u_{j}=\sum_{m \in \mathbb{Z}} G\left(j-m, \frac{t}{h^{2}}\right) u_{m}=\sum_{m \in \mathbb{Z}} G\left(m, \frac{t}{h^{2}}\right) u_{j-m}, \quad t \geq 0,
$$

where the semidiscrete heat kernel $G$ is defined as

$$
G(m, t)=e^{-2 t} I_{m}(2 t), \quad m \in \mathbb{Z}, t \geq 0 .
$$

Here $I_{\nu}$ is the modified Bessel function of order $\nu$ that satisfies

$$
\frac{\partial}{\partial t} I_{k}(t)=\frac{1}{2}\left(I_{k+1}(t)+I_{k-1}(t)\right)
$$

and from this we have immediately

$$
\frac{\partial}{\partial t}\left(e^{-2 t} I_{k}(2 t)\right)=e^{-2 t}\left(I_{k+1}(2 t)-2 I_{k}(2 t)+I_{k-1}(2 t)\right) .
$$

Then formula (2.1) for $h=1$ follows from (2.3). For a fixed $h \neq 1$, the formula (2.1) follows by scaling. See also [6, 11]. By (9.4) and 9.5) the kernel $G(m, t)$ is symmetric in $m$, that is, $G(m, t)=G(-m, t)$, and positive.

Let us begin now with the proof of Theorem 1.1.

First we check that if $u \in \ell_{s}, 0 \leq s \leq 1$, then $e^{t \Delta_{h}} u_{j}$ is well defined. Indeed, if $N>0$, for fixed $t, h>0$, by using the asymptotic of the Bessel function for large order 9.9),

$$
\begin{aligned}
\sum_{|m|>N} G\left(m, \frac{t}{h^{2}}\right)\left|u_{j-m}\right| & \leq C e^{-2 t / h^{2}} \sum_{|m|>N} \frac{\left(e t / h^{2}\right)^{|m|}(1+|m-j|)^{1+2 s}}{|m|^{|m|+1 / 2}} \frac{\left|u_{m-j}\right|}{(1+|m-j|)^{1+2 s}} \\
& \leq C e^{-2 t / h^{2}} \sup _{|m|>N} \frac{\left(e t / h^{2}\right)^{|m|}(1+|m|+|j|)^{1+2 s}}{|m|^{|m|+1 / 2}}\|u\|_{\ell_{s}} \\
& =C_{t, h, s, N, j}\|u\|_{\ell_{s}}<\infty .
\end{aligned}
$$

Next we prove each of the items of the statement of Theorem 1.1. (a). Define

$$
K_{s}^{h}(m)=\frac{1}{|\Gamma(-s)|} \int_{0}^{\infty} G\left(m, \frac{t}{h^{2}}\right) \frac{d t}{t^{1+s}}=\frac{1}{h^{2 s}|\Gamma(-s)|} \int_{0}^{\infty} G(m, r) \frac{d r}{r^{1+s}},
$$

for $m \neq 0$, and $K_{s}^{h}(0)=0$. The symmetry of this kernel in $m$ follows from the symmetry of $G(m, t)$. Therefore it is enough to assume that $m \in \mathbb{N}$. To get formula (1.7), we use 9.10) with $c=2$ and $\nu=m$. On the other hand, it is easy to show that $e^{t_{h}} 1 \equiv 1$ (see for example 
[6] for the case $h=1)$. Hence, from (1.3) and (2.1),

$$
\begin{aligned}
\left(-\Delta_{h}\right)^{s} u_{j} & =\frac{1}{\Gamma(-s)} \int_{0}^{\infty} \sum_{m \neq j} G\left(j-m, \frac{t}{h^{2}}\right)\left(u_{m}-u_{j}\right) \frac{d t}{t^{1+s}} \\
& =\frac{1}{\Gamma(-s)} \sum_{m \neq j}\left(u_{m}-u_{j}\right) \int_{0}^{\infty} G\left(j-m, \frac{t}{h^{2}}\right) \frac{d t}{t^{1+s}} \\
& =\sum_{m \neq j}\left(u_{j}-u_{m}\right) K_{s}^{h}(j-m) .
\end{aligned}
$$

For the interchange of summation and integration in the second equality, consider the terms

$$
\int_{0}^{\infty} \sum_{m \neq j} G\left(j-m, \frac{t}{h^{2}}\right)\left|u_{m}\right| \frac{d t}{t^{1+s}}+\left|u_{j}\right| \int_{0}^{\infty} \sum_{m \neq j} G\left(j-m, \frac{t}{h^{2}}\right) \frac{d t}{t^{1+s}} .
$$

By using (1.8) we see that the first term above is bounded by $C_{s, h} \sum_{m \neq j}|m-j|^{-(1+2 s)}\left|u_{m}\right|$, which is finite for each $j$ because $u \in \ell_{s}$. For the second term we use again (1.8).

(b). The two sided estimate in (1.8) follows from the explicit formula for the kernel (1.7) and the properties of the Gamma function we prove in Subsection 9.1, Lemma 9.2.

(c). Observe that

$$
h^{2 s} K_{s}^{h}(m)=K_{s}^{1}(m), \quad m \neq 0
$$

We have

$$
h^{2 s}\left(-\Delta_{h}\right)^{s} u_{j}=u_{j} \sum_{m \neq j} K_{s}^{1}(j-m)-\sum_{m \neq j} K_{s}^{1}(j-m) u_{m}=: u_{j} T_{1}-T_{2} .
$$

We write $T_{1}=T_{1,1}+T_{1,2}($ see $(2.5)$ ), where

$$
T_{1,2}=\frac{1}{|\Gamma(-s)|} \sum_{m \neq j} \int_{1}^{\infty} G(j-m, t) \frac{d t}{t^{1+s}}=\frac{1}{|\Gamma(-s)|} \sum_{m \neq 0} \int_{1}^{\infty} G(m, t) \frac{d t}{t^{1+s}} .
$$

We are going to prove that $T_{1,1}$ and $T_{2}$ tend to zero, while $T_{1,2}$ tends to 1 , as $s \rightarrow 0^{+}$. Let us begin with $T_{1,2}$. By adding and subtracting the term $m=0$ in the sum and using (9.6), we get

$$
T_{1,2}=\frac{1}{|\Gamma(-s)|}\left(\frac{1}{s}-\int_{1}^{\infty} \frac{e^{-2 t} I_{0}(2 t)}{t^{1+s}} d t\right)
$$

By noticing that $|\Gamma(-s)| s=\Gamma(1-s)$ and that, by $(9.8)$, we have

$$
\frac{1}{|\Gamma(-s)|} \int_{1}^{\infty} \frac{e^{-2 t} I_{0}(2 t)}{t^{1+s}} d t \leq \frac{C}{|\Gamma(-s)|} \int_{1}^{\infty} t^{-1 / 2-1-s} d t=\frac{C}{|\Gamma(-s)|(1 / 2+s)}
$$

we get $T_{1,2} \rightarrow 1$ as $s \rightarrow 0^{+}$, as desired. Next we handle the other two terms $T_{1,1}$ and $T_{2}$. On one hand, by 9.7 ,

$$
T_{1,1} \sim \frac{1}{|\Gamma(-s)|} \sum_{m \neq 0} \frac{1}{\Gamma(|m|+1)} \int_{0}^{1} e^{-2 t} t^{|m|} \frac{d t}{t^{1+s}} \leq \frac{1}{|\Gamma(-s)|} \sum_{m \neq 0} \frac{1}{\Gamma(|m|+1)} \frac{1}{|m|-s},
$$

and the last quantity tends to 0 as $s \rightarrow 0^{+}$. On the other hand, for $T_{2}$, we use (1.7) to obtain

$$
\left|T_{2}\right| \leq \frac{C_{s}}{|\Gamma(-s)|} \sum_{m \neq j} \frac{\Gamma(|j-m|-s)}{\Gamma(|j-m|+1+s)}\left|u_{m}\right| \text {. }
$$

The constant $C_{s}$ remains bounded as $s \rightarrow 0^{+}$. Since $u \in \ell_{0}$, by dominated convergence, the sum above is bounded by $\|u\|_{\ell_{0}}$, for each $j \in \mathbb{Z}$, as $s \rightarrow 0^{+}$. Therefore $T_{2} \rightarrow 0$ as $s \rightarrow 0^{+}$. 
$(d)$. By using the symmetry of the kernel $K_{s}^{h}$ we can write

$$
h^{2 s}\left(-\Delta_{h}\right)^{s} u_{j}=S_{1}+S_{2}
$$

where (recall (2.6)

$$
S_{1}=K_{s}^{1}(1)\left(-u_{j+1}+2 u_{j}-u_{j-1}\right), \quad \text { and } \quad S_{2}=\sum_{|m|>1} K_{s}^{1}(m)\left(u_{j}-u_{j-m}\right) .
$$

Next we show that $K_{s}^{1}(1) \rightarrow 1$, while $S_{2} \rightarrow 0$, as $s \rightarrow 1^{-}$, which would give the conclusion. By (1.7) with $h=1$ we have

$$
\lim _{s \rightarrow 1^{-}} K_{s}^{1}(1)=\lim _{s \rightarrow 1^{-}} \frac{4^{s} \Gamma(1-s) \Gamma(1 / 2+s)}{\sqrt{\pi}|\Gamma(-s)| \Gamma(2+s)}=\lim _{s \rightarrow 1^{-}} \frac{4^{s} \Gamma(1 / 2+s)}{\sqrt{\pi} \Gamma(2+s)}=\frac{4 \Gamma(3 / 2)}{\sqrt{\pi} \Gamma(3)}=1 .
$$

On the other hand, by (1.7) with $h=1, S_{2}$ is bounded by

$$
2\|u\|_{\ell_{h}^{\infty}} \sum_{|m|>1} K_{s}^{1}(m) \leq 2\|u\|_{\ell^{\infty}} \frac{4^{s} \Gamma\left(\frac{1}{2}+s\right)}{\pi^{1 / 2}|\Gamma(-s)|} \sum_{|m|>1} \frac{\Gamma(|m|-s)}{\Gamma(|m|+1+s)},
$$

which goes to zero as $s \rightarrow 1^{-}$.

Remark 2.1. In [7, formula (5)] the following equivalent expression for the kernel of $\left(-\Delta_{1}\right)^{s}$ is presented: for $m \neq 0$,

$$
K_{s}^{1}(m)=\frac{(-1)^{m+1} \Gamma(2 s+1)}{\Gamma(1+s+m) \Gamma(1+s-m)} .
$$

Indeed, apply the duplication formula and Euler's reflection formula for the Gamma function to 1.7 .

\section{Proof of Theorem 1.3}

A function $f: \mathbb{Z}_{h} \rightarrow \mathbb{R}$ is in $\ell_{h}^{p}, 1 \leq p<\infty$ if

$$
\|f\|_{\ell_{h}^{p}}=\left(h \sum_{j \in \mathbb{Z}}\left|f_{j}\right|^{p}\right)^{1 / p}<\infty
$$

while $f \in \ell_{h}^{\infty}$ if

$$
\|f\|_{\ell_{h}^{\infty}}=\sup _{h j \in \mathbb{Z}_{h}}\left|f_{j}\right|<\infty
$$

Obviously $\ell_{h}^{p} \subset \ell_{h}^{q}$ if $1 \leq p \leq q \leq \infty$, with $\|f\|_{\ell_{h}^{q}} \leq h^{1 / q-1 / p}\|f\|_{\ell_{h}^{p}}$. The discrete Hölder's inequality takes the form

$$
\|f g\|_{\ell_{h}^{1}} \leq\|f\|_{\ell_{h}^{p}}\|g\|_{\ell_{h}^{p^{\prime}}}, \quad \text { for } 1 \leq p \leq \infty, \frac{1}{p}+\frac{1}{p^{\prime}}=1 .
$$

When $h=1$ we write $\ell^{p}=\ell_{1}^{p}=\ell^{p}(\mathbb{Z})$.

(a). Observe that if $f \in \ell_{-s}$ then the semigroup $e^{t \Delta_{h}} f_{j}$ is well defined, for each $h j \in \mathbb{Z}_{h}$. This follows from an analogous computation to that of (2.4). By writing down the semidiscrete heat kernel into 1.10 and using Fubini's theorem (which will be fully justified once we prove $(1.12$ and $(1.13)$ we see that 1.11 follows with

$$
K_{-s}^{h}(m)=\frac{1}{\Gamma(s)} \int_{0}^{\infty} G\left(m, \frac{t}{h^{2}}\right) \frac{d t}{t^{1-s}}=\frac{1}{h^{-2 s} \Gamma(s)} \int_{0}^{\infty} G(m, r) \frac{d r}{r^{1-s}} .
$$

To get 1.12 , we just use the expression in $(2.2)$ and formula 9.10$)$ in the integral above.

(b). The estimates in (1.13) and (1.14) follow from 1.12 and Lemma 9.2 . 
(c). We recall that Stein and Wainger showed in [27, Proposition (a)] that the operator

$$
I_{\lambda} g_{j}=\sum_{m \in \mathbb{Z}, m \neq 0} \frac{g_{j-m}}{|m|^{\lambda}}, \quad j \in \mathbb{Z}, 0<\lambda<1,
$$

acting on functions $g: \mathbb{Z} \rightarrow \mathbb{R}$, is bounded from $\ell^{r}$ into $\ell^{l}$, whenever $1 / l \leq 1 / r-1+\lambda$ and $1<r<l<\infty$. By using Minkoswki's inequality, the estimate for the kernel $K_{-s}^{h}(m)$ in (1.13), the boundedness of the operator $I_{\lambda}$ above with $0<\lambda=1-2 s<1$ and $r=p, l=q$ as in our statement, the inclusion $\ell^{p} \subset \ell^{q}$ and (3.1), we get

$$
\begin{aligned}
\left\|\left(-\Delta_{h}\right)^{-s} f\right\|_{\ell_{h}^{q}} & =\left(\sum_{j \in \mathbb{Z}}\left|\sum_{m \in \mathbb{Z}} K_{-s}^{h}(m) f_{j-m}\right|^{q}\right)^{1 / q} \\
& =\left(\sum_{j \in \mathbb{Z}}\left|\sum_{m \in \mathbb{Z}, m \neq 0} K_{-s}^{h}(m) f_{j-m}+K_{-s}^{h}(0) f_{j}\right|^{q}\right)^{1 / q} \\
& \leq\left(\sum_{j \in \mathbb{Z}}\left|\sum_{m \in \mathbb{Z}, m \neq 0} K_{-s}^{h}(m) f_{j-m}\right|^{q}\right)^{1 / q}+C_{s} h^{2 s}\left(\sum_{j \in \mathbb{Z}}\left|f_{j}\right|^{q}\right)^{1 / q} \\
& \leq C_{s} h^{2 s}\left(\sum_{j \in \mathbb{Z}}\left|\sum_{m \in \mathbb{Z}, m \neq 0} \frac{f_{j-m}}{|m|^{1-2 s}}\right|^{q}\right)^{1 / q}+C_{s} h^{2 s}\left(\sum_{j \in \mathbb{Z}}\left|f_{j}\right|^{p}\right)^{1 / p} \\
& \leq C_{p, q, s} h^{2 s}\left(\sum_{j \in \mathbb{Z}}\left|f_{j}\right|^{p}\right)^{1 / p}+C_{s} h^{2 s-1 / p}\|f\|_{\ell_{h}^{p}} \leq C_{p, q, s} h^{2 s-1 / p}\|f\|_{\ell_{h}^{p} .}
\end{aligned}
$$

Multiply both sides by $h^{1 / q}$ and recall 3.1 to reach 1.15 .

\section{Proof of Theorems 1.5 and 1.7}

For the reader's convenience, we recall the definition of Hölder spaces on the real line.

Definition 4.1 (Continuous Hölder spaces). Given $k \in \mathbb{N}_{0}$ and $0<\alpha \leq 1$, we say that a continuous function $U: \mathbb{R} \rightarrow \mathbb{R}$ belongs to the Hölder space $C^{k, \alpha}$ if $U \in C^{k}$ and

$$
[U]_{C^{k, \alpha}} \equiv\left[U^{(k)}\right]_{C^{0, \alpha}}:=\sup _{\substack{x, y \in \mathbb{R} \\ x \neq y}} \frac{\left|U^{(k)}(x)-U^{(k)}(y)\right|}{|x-y|^{\alpha}}<\infty,
$$

where $U^{(k)}$ denotes the $k$-th derivative of $U$. The norm in the spaces $C^{k, \alpha}$ is given by

$$
\|U\|_{C^{k, \alpha}}:=\sum_{l=0}^{k}\left\|U^{(l)}\right\|_{L^{\infty}}+\left[U^{(k)}\right]_{C^{0, \alpha}} .
$$

Next we define the discrete Hölder spaces on the mesh $\mathbb{Z}_{h}$. For $u: \mathbb{Z}_{h} \rightarrow \mathbb{R}$ we consider the first order difference operators

$$
D_{+} u_{j}=\frac{1}{h}\left(u_{j+1}-u_{j}\right), \quad \text { and } \quad D_{-} u_{j}=\frac{1}{h}\left(u_{j}-u_{j-1}\right) .
$$

For $\gamma, \eta \in \mathbb{N}_{0}$, let $D_{+,-}^{\gamma, \eta} u_{j}:=D_{+}^{\gamma} D_{-}^{\eta} u_{j}$, where $D_{ \pm}^{k} u$ means that we apply $k$-times the operator $D_{ \pm}$to $u$, with $D_{ \pm}^{0} u=u$. 
Definition 4.2 (Discrete Hölder spaces). Let $k \in \mathbb{N}_{0}$ and $0<\alpha \leq 1$. A function $u: \mathbb{Z}_{h} \rightarrow \mathbb{R}$ belongs to the discrete Hölder space $C_{h}^{k, \alpha}$ if there is a constant $C>0$ such that

$$
[u]_{C_{h}^{k, \alpha}} \equiv\left[D_{+,-}^{\gamma, \eta} u\right]_{C_{h}^{0, \alpha}}:=\sum_{\gamma, \eta: \gamma+\eta=k} \sup _{\substack{h m, h j \in \mathbb{Z}_{h} \\ m \neq j}} \frac{\left|D_{+,-}^{\gamma, \eta} u_{j}-D_{+,-}^{\gamma, \eta} u_{m}\right|}{|h j-h m|^{\alpha}} \leq C<\infty .
$$

Remark 4.3. It is obvious that if $u: \mathbb{Z}_{h} \rightarrow \mathbb{R}$ is bounded then it belongs to $C_{h}^{0, \alpha}$, for any $0<\alpha \leq 1$. In this case a discrete Hölder norm can be given by $\|u\|_{C_{h}^{0, \alpha}}:=\|u\|_{\ell_{h}^{\infty}}+[u]_{C_{h}^{0, \alpha}}$.

\subsection{Proof of Theorem $\mathbf{1 . 5}$.}

(i). It suffices to prove the case $k=0$, since $D_{ \pm}$commutes with $\left(-\Delta_{h}\right)^{s}$. Let $h k, h j \in \mathbb{Z}_{h}$. By recalling (2.6), we can write

$$
\left|\left(-\Delta_{h}\right)^{s} u_{k}-\left(-\Delta_{h}\right)^{s} u_{j}\right|=\frac{1}{h^{2 s}}\left|S_{1}+S_{2}\right|,
$$

where

$$
S_{1}:=\sum_{1 \leq|m| \leq|k-j|}\left(u_{k}-u_{k+m}-u_{j}+u_{j+m}\right) K_{s}^{1}(m),
$$

and $S_{2}$ is the rest of the sum over $|m|>|k-j|$. By the kernel estimate (1.8),

$$
S_{1} \leq C_{s} 2[u]_{C_{h}^{0, \alpha}} h^{\alpha} \sum_{1 \leq|m| \leq|k-j|} \frac{|m|^{\alpha}}{|m|^{1+2 s}} \leq C_{s}[u]_{C_{h}^{0, \alpha}} h^{\alpha}|k-j|^{\alpha-2 s} .
$$

For $S_{2}$ we use that $\left|u_{k}-u_{j}\right| \leq[u]_{C_{h}^{0, \alpha}} h^{\alpha}|k-j|^{\alpha}$ and (1.8) again to get

$$
S_{2} \leq C_{s}[u]_{C_{h}^{\alpha}} h^{\alpha}|k-j|^{\alpha} \sum_{|m|>|k-j|}|m|^{-1-2 s} \leq C_{s}[u]_{C_{h}^{0, \alpha}} h^{\alpha}|k-j|^{\alpha-2 s} .
$$

We conclude by pasting together both estimates into (4.2).

(ii). As in $(i)$, it is enough to consider just the case $k=0$. We are going to use (4.2). Without loss of generality, let $m \in \mathbb{N}$. We split the sum in $(4.2)-(4.3)$ by taking the terms $u_{k}-u_{k+m}$ and $u_{j}-u_{j+m}$ separately. The following computation works for both terms, so we do it only for the first one. It is verified that

$$
u_{k+m}-u_{k}=h \sum_{\gamma=0}^{m-1} D_{+} u_{k+\gamma} .
$$

Therefore,

$$
u_{k}-u_{k+m}=\left(h m D_{+} u_{k}-h \sum_{\gamma=0}^{m-1} D_{+} u_{k+\gamma}\right)-h m D_{+} u_{k}
$$

On one hand, by taking into account that the kernel $K_{s}^{1}(m)$ is even, we get

$$
\sum_{1 \leq|m| \leq|k-j|}\left(h m D_{+} u_{k}\right) K_{s}^{1}(m)=h D_{+} u_{k} \sum_{1 \leq|m| \leq|k-j|} m K_{s}^{1}(m)=0 .
$$


On the other hand, since $u \in C_{h}^{1, \alpha}$, the first term in the right hand side of 4.5 can be bounded by

$$
\begin{aligned}
h \sum_{\gamma=0}^{m-1}\left|D_{+} u_{k}-D_{+} u_{k+\gamma}\right| & \leq h^{1+\alpha}[u]_{C_{h}^{1, \alpha}} \sum_{\gamma=0}^{m-1}|\gamma|^{\alpha} \\
& \leq h^{1+\alpha}[u]_{C_{h}^{1, \alpha}}|m|^{\alpha}|m|=[u]_{C_{h}^{1, \alpha}}(h|m|)^{1+\alpha} .
\end{aligned}
$$

Using (4.6) and (4.7) (and their analogous for $u_{j}-u_{j+m}$ ) in (4.3), we conclude that

$$
\left|S_{1}\right| \leq C_{s}[u]_{C_{h}^{1, \alpha}} h^{1+\alpha} \sum_{1 \leq|m| \leq|k-j|} \frac{|m|^{1+\alpha}}{|m|^{1+2 s}} \leq C_{s}[u]_{C_{h}^{1, \alpha}}(h|k-j|)^{1+\alpha-2 s} .
$$

Now we deal with $S_{2}$. By 4.4 ,

$$
\begin{aligned}
\left|\left(u_{k}-u_{j}\right)-\left(u_{k+m}-u_{j+m}\right)\right| & =\left|\left(u_{(k-j)+j}-u_{j}\right)-\left(u_{(k-j)+(j+m)}-u_{j+m}\right)\right| \\
& \leq h \sum_{\gamma=0}^{k-j-1}\left|D_{+} u_{j+\gamma}-D_{+} u_{j+m+\gamma}\right| \leq[u]_{C_{h}^{1, \alpha}} h^{1+\alpha}|m|^{\alpha}|k-j| .
\end{aligned}
$$

Hence,

$$
\left|S_{2}\right| \leq C[u]_{C_{h}^{1, \alpha}} h^{1+\alpha}|k-j| \sum_{|m|>|k-j|}|m|^{\alpha} K_{s}^{1}(m) \leq C[u]_{C_{h}^{1, \alpha}} h^{1+\alpha}|k-j|^{1+\alpha-2 s} .
$$

4.2. Proof of Theorem 1.7. We need a preliminary lemma.

Lemma 4.4. Let $0<s<1$ and let $A_{s}>0$ be as in (1.9). Given $j \in \mathbb{Z}$, we have

$$
\begin{gathered}
\left|A_{s} \int_{|y-h(j+m)|<h / 2} \frac{d y}{|h j-y|^{1+2 s}}-K_{s}^{h}(m)\right| \leq \frac{C_{s}}{h^{2 s}|m|^{2+2 s}}, \quad \text { for all } m \in \mathbb{Z} \backslash\{0\}, \\
\int_{|y-h(j+m)|<h / 2} \frac{d y}{|h j-y|^{1+2 s}} \leq \frac{C_{s}}{h^{2 s}|m|^{1+2 s}}, \quad \text { for all } m \in \mathbb{Z} \backslash\{0\},
\end{gathered}
$$

and

$$
\sum_{m \in \mathbb{Z}} \int_{|y-h(j+m)|<h / 2} \frac{h j-y}{|h j-y|^{1+2 s}} d y=0 .
$$

Proof. Let $m \in \mathbb{Z} \backslash\{0\}$. The change of variable $h j-y=h z$ and 2.6 produce

$$
\begin{aligned}
& \left|\frac{A_{s}}{h^{2 s}} \int_{|z-m|<1 / 2} \frac{d z}{|z|^{1+2 s}}-K_{s}^{h}(m)\right| \\
& \quad \leq\left|\frac{A_{s}}{h^{2 s}} \int_{|z-m|<1 / 2}\left(\frac{1}{|z|^{1+2 s}}-\frac{1}{|m|^{1+2 s}}\right) d z\right|+h^{-2 s}\left|\frac{A_{s}}{|m|^{1+2 s}}-K_{s}^{1}(m)\right| .
\end{aligned}
$$

By using the mean value theorem,

$$
\left|\int_{|z-m|<1 / 2}\left(\frac{1}{|z|^{1+2 s}}-\frac{1}{|m|^{1+2 s}}\right) d z\right| \leq C_{s}\left|\int_{|z-m|<1 / 2} \frac{d z}{|m|^{2+2 s}}\right|=\frac{C_{s}}{|m|^{2+2 s}},
$$

while by Lemma 9.2 ,

$$
\left|\frac{A_{s}}{|m|^{1+2 s}}-K_{s}^{1}(m)\right| \leq \frac{C_{s}}{|m|^{2+2 s}}
$$


Thus (4.8) follows. For (4.9), it is easy to see that

$$
\int_{|y-(h(j+m))|<h / 2} \frac{d y}{|h j-y|^{1+2 s}} \leq C_{s} \int_{|y-(h(j+m))|<h / 2} \frac{d y}{|h m|^{1+2 s}}=\frac{C_{s}}{h^{2 s}|m|^{1+2 s}} .
$$

Finally, let us prove 4.10). By symmetry, we have

$$
\int_{|y-h j|<h / 2} \frac{(h j-y)}{|h j-y|^{1+2 s}} d y=0 .
$$

Moreover, by changing variables $h j-y=z$, we get

$$
\sum_{\substack{m \in \mathbb{Z} \\ m \neq 0}} \int_{|z-h m|<h / 2} \frac{z}{|z|^{1+2 s}} d z=\sum_{\substack{\ell \in \mathbb{Z} \\ \ell \neq 0}} \int_{|z+h \ell|<h / 2} \frac{z}{|z|^{1+2 s}} d z=\sum_{\substack{\ell \in \mathbb{Z} \\ \ell \neq 0}} \int_{|r-h \ell|<h / 2} \frac{-r}{|r|^{1+2 s}} d r,
$$

and the conclusion readily follows.

Now we present the proof of Theorem 1.7 .

(i). We write, for each $j \in \mathbb{Z}$,

$$
\begin{aligned}
&\left(r_{h}\left((-\Delta)^{s} U\right)\right)_{j}= A_{s} \sum_{m \in \mathbb{Z}} \int_{|y-h(j+m)|<h / 2} \frac{U(h j)-U(y)}{|h j-y|^{1+2 s}} d y \\
&=A_{s}\left[\int_{|y-h j|<h / 2} \frac{U(h j)-U(y)}{|h j-y|^{1+2 s}} d y\right. \\
&+\sum_{\substack{m \in \mathbb{Z} \\
m \neq 0}} \int_{|y-h(j+m)|<h / 2} \frac{U(h(j+m))-U(y)}{|h j-y|^{1+2 s}} d y \\
&\left.+\sum_{\substack{m \in \mathbb{Z} \\
m \neq 0}}(U(h j)-U(h(j+m))) \int_{|y-h(j+m)|<h / 2} \frac{d y}{|h j-y|^{1+2 s}}\right] \\
&=: A_{s}\left(S_{0}+S_{1}+S_{2}\right) .
\end{aligned}
$$

We readily notice that

$$
\left|S_{0}\right| \leq[U]_{C^{0, \alpha}} \int_{|h j-y| \leq h / 2}|h j-y|^{\alpha-2 s-1} d y \leq C_{s}[U]_{C^{0, \alpha}} h^{\alpha-2 s}
$$

By using that $U \in C^{0, \alpha}$ and 4.9 , we have

$$
\begin{aligned}
\left|S_{1}\right| & \leq C[U]_{C^{0, \alpha}} \sum_{\substack{m \in \mathbb{Z} \\
m \neq 0}} \int_{|y-h(j+m)|<h / 2} \frac{h^{\alpha} d y}{|h j-y|^{1+2 s}} \\
& \leq C_{s}[U]_{C^{0, \alpha}} h^{\alpha} \sum_{\substack{m \in \mathbb{Z} \\
m \neq 0}} \frac{1}{h^{2 s}|m|^{1+2 s}}=C_{s}[U]_{C^{0, \alpha}} h^{\alpha-2 s} .
\end{aligned}
$$


Now we compare $A_{s} S_{2}$ with $\left(-\Delta_{h}\right)^{s}\left(r_{h} U\right)_{j}$. Since $U \in C^{0, \alpha}$, by Lemma 4.4 we can see that

$$
\begin{gathered}
\left|A_{s} \sum_{\substack{m \in \mathbb{Z} \\
m \neq 0}}(U(h j)-U(h(j+m))) \int_{|y-h(j+m)|<h / 2} \frac{d y}{|h j-y|^{1+2 s}}-\left(-\Delta_{h}\right)^{s}\left(r_{h} U\right)_{j}\right| \\
\quad \leq \sum_{\substack{m \in \mathbb{Z} \\
m \neq 0}}|U(h j)-U(h(j+m))|\left|A_{s} \int_{|y-h(j+m)|<h / 2} \frac{d y}{|h j-y|^{1+2 s}}-K_{s}^{h}(m)\right| \\
\leq C_{s}[U]_{C^{0, \alpha}} \sum_{\substack{m \in \mathbb{Z} \\
m \neq 0}} \frac{|h m|^{\alpha}}{h^{2 s}|m|^{2+2 s}} \leq C_{s}[U]_{C^{0, \alpha}} h^{\alpha-2 s} .
\end{gathered}
$$

(ii). Observe that $\frac{d}{d x}$ and $D_{+}$commute with $(-\Delta)^{s}$ and $\left(-\Delta_{h}\right)^{s}$, respectively. Then

$$
\begin{aligned}
& \left\|D_{+}\left(-\Delta_{h}\right)^{s}\left(r_{h} U\right)-r_{h}\left(\frac{d}{d x}(-\Delta)^{s} U\right)\right\|_{\ell^{\infty}} \\
& \quad \leq\left\|\left(-\Delta_{h}\right)^{s} D_{+}\left(r_{h} U\right)-\left(-\Delta_{h}\right)^{s}\left(r_{h} \frac{d}{d x} U\right)\right\|_{\ell^{\infty}}+\left\|\left(-\Delta_{h}\right)^{s}\left(r_{h} \frac{d}{d x} U\right)-r_{h}\left(\frac{d}{d x}(-\Delta)^{s} U\right)\right\|_{\ell^{\infty}} .
\end{aligned}
$$

For the second term we just apply $(i)$. As for the first one, by using the mean value theorem,

$$
\begin{aligned}
& \mid\left(-\Delta_{h}\right)^{s} D_{+}\left(r_{h} U\right)_{j}-\left(-\Delta_{h}\right)^{s}\left(r_{h} \frac{d}{d x} U\right)_{j} \mid \\
&= \mid \sum_{\substack{m \in \mathbb{Z} \\
m \neq 0}} K_{s}^{h}(m)\left[\left(\frac{U(h(j+1))-U(h j)}{h}-U^{\prime}(h j)\right)\right. \\
&\left.\quad-\left(\frac{U(h(j+m+1))-U(h(j+m)))}{h}-U^{\prime}(h(j+m))\right)\right] \mid \\
&=\left|\sum_{\substack{m \in \mathbb{Z} \\
m \neq 0}} K_{s}^{h}(m)\left[\left(U^{\prime}\left(\xi_{j}\right)-U^{\prime}(h j)\right)-\left(U^{\prime}\left(\xi_{j+m}\right)-U^{\prime}(h(j+m))\right)\right]\right| \\
& \leq C[U]_{C^{1, \alpha}} \sum_{\substack{m \in \mathbb{Z} \\
m \neq 0}} K_{s}^{h}(m) h^{\alpha} \leq C[U]_{C^{1, \alpha}} h^{\alpha-2 s},
\end{aligned}
$$

where $\xi_{j}$ is an intermediate point between $h j$ and $h(j+1)$, and analogously $\xi_{j+m}$. (iii). By taking into account (4.10), we can write

$$
\begin{aligned}
& r_{h}\left((-\Delta)^{s} U\right)_{j}=A_{s} \sum_{m \in \mathbb{Z}} \int_{|y-h(j+m)|<h / 2} \frac{U(h j)-U(y)-U^{\prime}(h j)(h j-y)}{|h j-y|^{1+2 s}} d y \\
& =A_{s}\left[\int_{|y-h j|<h / 2} \frac{U(h j)-U(y)-U^{\prime}(h j)(h j-y)}{|h j-y|^{1+2 s}} d y\right. \\
& \quad+\sum_{\substack{m \in \mathbb{Z} \\
m \neq 0}} \int_{|y-h(j+m)|<h / 2} \frac{U(h(j+m))-U(y)-U^{\prime}(h j)(h(j+m)-y)}{|h j-y|^{1+2 s}} d y \\
& \left.\quad+\sum_{\substack{m \in \mathbb{Z} \\
m \neq 0}}\left(U(h j)-U(h(j+m))-U^{\prime}(h j)(h j-h(j+m))\right) \int_{|y-h(j+m)|<h / 2} \frac{d y}{|h j-y|^{1+2 s}}\right] \\
& =: A_{s}\left(T_{0}+T_{1}+T_{2}\right) .
\end{aligned}
$$


For $T_{0}$, we use the mean value theorem and the hypothesis on $U$. Indeed, if $|y-h j|<h / 2$ and $\xi_{j}(y)$ is an intermediate point between $h j$ and $y$, we have

$$
\begin{aligned}
\frac{\left(U^{\prime}\left(\xi_{j}(y)\right)-U^{\prime}(h j)\right)(h j-y)}{|h j-y|^{1+2 s}} & \leq[U]_{C^{1, \alpha}} \frac{\left|\xi_{j}(y)-h j\right|^{\alpha}|h j-y|}{|h j-y|^{1+2 s}} \\
& \leq[U]_{C^{1, \alpha}}|h j-y|^{\alpha-2 s} .
\end{aligned}
$$

Then, as a consequence,

$$
\left|T_{0}\right| \leq[U]_{C^{1, \alpha}} \int_{|y-h j|<h / 2}|h j-y|^{\alpha-2 s} d y \leq C_{s}[U]_{C^{1, \alpha}} h^{\alpha-2 s+1},
$$

whenever $2 s<1+\alpha$. By the hypotheses and 4.9 ),

$$
\left|T_{1}\right| \leq C_{s}[U]_{C^{1, \alpha}} \sum_{\substack{m \in \mathbb{Z} \\ m \neq 0}} \frac{|h m|^{\alpha} h}{h^{2 s}|m|^{1+2 s}}=C_{s}[U]_{C^{1, \alpha}} h^{1+\alpha-2 s} .
$$

We compare $A_{s} T_{2}$ with $\left(-\Delta_{h}\right)^{s}\left(r_{h} U\right)_{j}$. Since $K_{s}^{h}(m)$ is even in $m$, we can write

$$
\left(-\Delta_{h}\right)^{s}\left(r_{h} U\right)_{j}=\sum_{\substack{m \in \mathbb{Z} \\ m \neq 0}}\left(U(h j)-U(h(j+m))-U^{\prime}(h j)(h j-h(j+m))\right) K_{s}^{h}(m) .
$$

Then (4.8) and the regularity of $U$ give the result.

(iv). The proof in this case follows as in (ii) by iteration $l$ times.

\section{Proof of Theorem 1.6}

We shall need two lemmas.

Lemma 5.1. Let $0<s<1 / 2$ and $H_{s}(r):=\int_{0}^{\infty} e^{-(r+s) v}\left(1-e^{-v}\right)^{-2 s} d v$, for $r>0$. For any $k \geq 0$ there exists a constant $C_{k, s}>0$ such that

$$
\left|\frac{d^{k}}{d r^{k}} H_{s}(r)\right| \leq \frac{C_{k, s}}{(r+s)^{k+1-2 s}}, \quad \text { for all } r>0 .
$$

Proof. We have

$$
\begin{aligned}
\left|\frac{d^{k}}{d r^{k}} H_{s}(r)\right| & =\left|(-1)^{k} \int_{0}^{\infty} e^{-(r+s) v}\left(1-e^{-v}\right)^{-2 s} v^{k} d v\right| \\
& =\int_{0}^{1}+\int_{1}^{\infty} e^{-(r+s) v}\left(1-e^{-v}\right)^{-2 s} v^{k} d v=: I_{1}+I_{2} .
\end{aligned}
$$

On one hand, since $1-e^{-v} \geq\left(1-e^{-1}\right) v=C v$ for $v \in(0,1)$,

$$
\begin{aligned}
I_{1} \leq C_{s} \int_{0}^{1} e^{-(r+s) v} v^{k-2 s} d v & =C_{s} \int_{0}^{r+s} e^{-t} \frac{t^{k-2 s}}{(r+s)^{k+1-2 s}} d t \\
& \leq \frac{C_{s}}{(r+s)^{k+1-2 s}} \int_{0}^{\infty} e^{-t} t^{k-2 s} d t \\
& =C_{s} \frac{\Gamma(k+1-2 s)}{(r+s)^{k+1-2 s}} .
\end{aligned}
$$

On the other hand,

$$
I_{2} \leq \int_{1}^{\infty} e^{-(r+s) v} v^{k} d v=(r+s)^{-(k+1)} \int_{r+s}^{\infty} e^{-t} t^{k} d t \leq \frac{\Gamma(k+1)}{(r+s)^{k+1}} .
$$


By collecting both estimates, we conclude that

$$
\left|\frac{d^{k}}{d r^{k}} H_{s}(r)\right| \leq C_{s} \frac{\Gamma(k+1-2 s)}{(r+s)^{k+1-2 s}}+\frac{\Gamma(k+1)}{(r+s)^{k+1}} \leq \frac{C_{k, s}}{(r+s)^{k+1-2 s}},
$$

because $(r+s)^{-(k+1)} \leq C_{s}(r+s)^{-(k+1-2 s)}$.

Recall the identity for the quotient of Gamma functions in [30, Section 7 (15)]:

$$
\frac{\Gamma(z+\alpha)}{\Gamma(z+\beta)}=\frac{1}{\Gamma(\beta-\alpha)} \int_{0}^{\infty} e^{-(z+\alpha) v}\left(1-e^{-v}\right)^{\beta-\alpha-1} d v,
$$

valid for $\operatorname{Re}(\beta-\alpha)>0, \operatorname{Re}(z+\alpha)>0$. It follows from 1.12 and (5.1) with $z=|m|$, $\alpha=s \in(0,1 / 2)$ and $\beta=1-s$, that

$$
K_{-s}^{h}(m)=C_{s} h^{2 s} H_{s}(|m|), \quad m \in \mathbb{Z},
$$

for some constant $C_{s}>0$, where $H_{s}$ is the function we defined in Lemma 5.1.

Lemma 5.2. Let $0<s<1 / 2$ and $j, k \in \mathbb{Z}$. Then

$$
\sum_{m \in \mathbb{Z}}\left(K_{-s}^{h}(m-j)-K_{-s}^{h}(m-k)\right)=0 .
$$

Proof. Clearly it is enough to prove that for every positive integer $j$ we have

$$
\sum_{m \in \mathbb{Z}}\left(K_{-s}^{1}(m-j)-K_{-s}^{1}(m)\right)=0,
$$

where

$$
K_{-s}^{1}(m)=h^{-2 s} K_{s}^{h}(m), \quad m \in \mathbb{Z} .
$$

Observe that by Lemma 5.1 the series above is absolutely convergent. On the other hand, by the symmetry of the kernel $K_{-s}^{1}$ we have

$$
\sum_{m<0}\left(K_{-s}^{1}(m-j)-K_{-s}^{1}(m)\right)=\sum_{m>0}\left(K_{-s}^{1}(m+j)-K_{-s}^{1}(m)\right),
$$

and

$$
\sum_{m>j}\left(K_{-s}^{1}(m-j)-K_{-s}^{1}(m)\right)=\sum_{m>0}\left(K_{-s}^{1}(m)-K_{-s}^{1}(m+j)\right) .
$$

Finally,

$$
\begin{aligned}
\sum_{0 \leq m \leq j}\left(K_{-s}^{1}(m-j)-K_{-s}^{1}(m)\right)= & K_{-s}^{1}(-j)-K_{-s}^{1}(0)+K_{-s}^{1}(1-j)-K_{-s}^{1}(1) \\
& +\cdots+K_{-s}^{1}(-1)-K_{-s}^{1}(j-1)+K_{-s}^{1}(0)-K_{-s}^{1}(j) \\
= & 0 .
\end{aligned}
$$

Pasting together these computations we get the claim.

We are ready to begin with the proof of Theorem 1.6. We shall prove the result only for the case $h=1$. The general case $h>0$ follows by using the relation $(5.3)$.

(i). As the discrete derivatives commute with $\left(-\Delta_{1}\right)^{-s}$ for $0<s<1 / 2$, it suffices to prove the case $k=0$. Moreover, it is enough to show that for any positive $j$ we have

$$
\left|\left(-\Delta_{1}\right)^{-s} f_{j}-\left(-\Delta_{1}\right)^{-s} f_{0}\right| \leq C[f]_{C_{1}^{0, \alpha}} j^{\alpha+2 s} .
$$


By using Lemma 5.2 we can write

$$
\begin{aligned}
\left|\left(-\Delta_{1}\right)^{-s} f_{j}-\left(-\Delta_{1}\right)^{-s} f_{0}\right| & =\left|\sum_{m \in \mathbb{Z}}\left(K_{-s}^{1}(m-j)-K_{-s}^{1}(m)\right)\left(f_{m}-f_{0}\right)\right| \\
& \leq\left(\sum_{0<|m| \leq 2 j}+\sum_{|m|>2 j}\right)\left|K_{-s}^{1}(m-j)-K_{-s}^{1}(m)\right|\left|f_{m}-f_{0}\right| \\
& =: S_{1}+S_{2} .
\end{aligned}
$$

By using the estimate in 1.13 we get

$$
\begin{aligned}
S_{1} & \leq C[f]_{C_{1}^{0, \alpha}}\left(K_{-s}^{1}(0) j^{\alpha}+\sum_{0<|m| \leq 2 j, m \neq j} \frac{|m|^{\alpha}}{|m-j|^{1-2 s}}+\sum_{0<|m| \leq 2 j} \frac{|m|^{\alpha}}{|m|^{1-2 s}}\right) \\
& \leq C[f]_{C_{1}^{0, \alpha}} j^{\alpha}\left(1+\sum_{0<|m-j| \leq 3 j} \frac{1}{|m-j|^{1-2 s}}+\sum_{0<|m| \leq 2 j} \frac{1}{|m|^{1-2 s}}\right) \\
& \leq C[f]_{C_{1}^{0, \alpha}} j^{\alpha+2 s} .
\end{aligned}
$$

On the other hand, $(5.2)$, the mean value theorem and Lemma 5.1 with $k=1$ allow us to estimate

$$
\begin{aligned}
S_{2} & \leq C[f]_{C_{1}^{0, \alpha}} \sum_{|m|>2 j}\left|H_{s}(|m-j|)-H_{s}(|m|)\right||m|^{\alpha} \\
& \leq C[f]_{C_{1}^{0, \alpha}} j \sum_{|m|>j} \frac{|m|^{\alpha}}{|m|^{2-2 s}} \leq C[f]_{C_{1}^{0, \alpha}} j^{\alpha+2 s} .
\end{aligned}
$$

(ii). Again, it is enough to prove only the case $k=0$. By Lemma 5.2 ,

$$
\begin{aligned}
D_{+} & \left(\left(-\Delta_{1}\right)^{-s} f_{j}\right)-D_{+}\left(\left(-\Delta_{1}\right)^{-s} f_{0}\right) \\
& =\left(\left(-\Delta_{1}\right)^{-s} f_{j+1}-\left(-\Delta_{1}\right)^{-s} f_{j}\right)-\left(\left(-\Delta_{1}\right)^{-s} f_{1}-\left(-\Delta_{1}\right)^{-s} f_{0}\right) \\
& =\sum_{|m|>0}\left[\left(K_{-s}^{1}(m-(j+1))-K_{-s}^{1}(m-j)\right)-\left(K_{-s}^{1}(m-1)-K_{-s}^{1}(m)\right)\right]\left(f_{m}-f_{0}\right) .
\end{aligned}
$$

Proceeding as in $(i)$, we decompose into the sums $T_{1}=\sum_{0<|m| \leq 2 j}$ and $T_{2}=\sum_{|m|>2 j}$. To estimate $T_{1}$, we use the estimates

$$
\left|K_{-s}^{1}(m-1)-K_{-s}^{1}(m)\right| \leq \frac{C_{s}}{|m|^{2-2 s}}, \quad m \neq 0,
$$

and

$$
\left|K_{-s}(m-(j+1))-K_{-s}(m-j)\right| \leq \frac{C_{s}}{|m-j|^{2-2 s}}, \quad m \neq j .
$$

They are deduced from $(5.2)$, the mean value theorem and Lemma 5.1 with $k=1$ for $m \neq 1$ and $m \neq j+1$, respectively. The particular cases $m=1$ and $m=j+1$ are trivial.

Then, by observing that $2 s<1$, we can proceed as in $(i)$, arriving at $T_{1} \leq C[f]_{C_{1}^{0, \alpha}} j^{\alpha+2 s-1}$. Regarding the term $T_{2}$, we write, up to a multiplicative constant depending on $s$ (see 5.2 ),

$$
\begin{aligned}
&\left(K_{-s}^{1}(m-(j+1))-K_{-s}^{1}(m-j)\right)-\left(K_{-s}^{1}(m-1)-K_{-s}^{1}(m)\right) \\
&=H_{s}(|m-(j+1)|)-H_{s}(|m-j|)-\left(H_{s}(|m-1|)-H_{s}(|m|)\right) .
\end{aligned}
$$


By a repeated application of the mean value theorem and Lemma 5.1 with $k=2$ we then get

$$
\left|\left(K_{-s}^{1}(m-(j+1))-K_{-s}^{1}(m-j)\right)-\left(K_{-s}^{1}(m-1)-K_{-s}^{1}(m)\right)\right| \leq C_{s} \frac{j}{|m|^{3-2 s}} .
$$

Hence

$$
T_{2} \leq C[f]_{C_{1}^{0, \alpha}} j \sum_{|m|>j} \frac{|m|^{\alpha}}{|m|^{3-2 s}} \leq C[f]_{C_{1}^{0, \alpha}} j^{\alpha+2 s-1} .
$$

(iii). The proof of $(i)$ can be adapted to this case, details are left to the interested reader.

\section{The Dirichlet problem for the fractional Discrete Laplacian}

Throughout this section we fix a finite interval $B^{h} \subset \mathbb{Z}_{h}$. The aim of this section is to show the following existence and uniqueness result.

Theorem 6.1 (Discrete Dirichlet problem). Let $0<s<1 / 2$ and $f: B^{h} \rightarrow \mathbb{R}$. Then there exists a unique solution $u: \mathbb{Z}_{h} \rightarrow \mathbb{R}$ to the nonlocal discrete Dirichlet problem

$$
\begin{cases}\left(-\Delta_{h}\right)^{s} u=f, & \text { in } B^{h}, \\ u=0, & \text { in } \mathbb{Z}_{h} \backslash B^{h} .\end{cases}
$$

Before presenting the proof we need some preliminaries.

We first observe that if $u: \mathbb{Z}_{h} \rightarrow \mathbb{R}$ is a bounded function then $\left(-\Delta_{h}\right)^{s} u$ is well defined and bounded, with

$$
h^{2 s}\left\|\left(-\Delta_{h}\right)^{s} u\right\|_{\ell_{h}^{\infty}} \leq C_{s}\|u\|_{\ell_{h}^{\infty}} .
$$

Indeed, for any $h j \in \mathbb{Z}_{h}$, by (1.6) and 1.8 ,

$$
\left|\left(-\Delta_{h}\right)^{s} u_{j}\right| \leq \frac{C_{s}}{h^{2 s}} \sum_{m \neq j} \frac{2\|u\|_{\ell_{h}^{\infty}}}{|j-m|^{1+2 s}}=\frac{C_{s}}{h^{2 s}}\|u\|_{\ell_{h}^{\infty}} \sum_{m \neq 0} \frac{1}{|m|^{1+2 s}} .
$$

In particular, $\left(-\Delta_{h}\right)^{s} u$ is a well defined bounded function whenever $u \in \ell_{h}^{p}$, for any $1 \leq p \leq$ $\infty$. We also observe that, for $0<s<1$,

$$
\text { if } u \in \ell_{h}^{2} \text { then }\left(-\Delta_{h}\right)^{s} u \in \ell_{h}^{2} \text {, with }\left\|\left(-\Delta_{h}\right)^{s} u\right\|_{\ell_{h}^{2}} \leq \frac{4^{s}}{h^{2 s}}\|u\|_{\ell_{h}^{2}} .
$$

This follows, for example, by using the Fourier transform, which we now introduce. Let $\mathbb{T}_{h}=\mathbb{R} /\left(2 \pi \mathbb{Z}_{h}\right)=\mathbb{R} /(2 \pi h \mathbb{Z})$, the one dimensional torus of length $2 \pi h$, which we identify with the interval $[-h \pi, h \pi)$. We denote $L_{h}^{2}=L^{2}\left(\mathbb{T}_{h}\right)$ with inner product

$$
\langle U, V\rangle_{L_{h}^{2}}=\int_{-h \pi}^{h \pi} U(\theta) \overline{V(\theta)} d \theta
$$

Then the set of exponentials $\left\{(2 \pi h)^{-1 / 2} e^{i j \theta / h}: j \in \mathbb{Z}, \theta \in \mathbb{T}_{h}\right\}$, where $i$ denotes the imaginary unit, forms an orthonormal basis of $L_{h}^{2}$. For an integrable function $U: \mathbb{T}_{h} \rightarrow \mathbb{R}$, its Fourier series is given by

$$
S[U](\theta)=\frac{1}{(2 \pi h)^{1 / 2}} \sum_{j \in \mathbb{Z}} \widehat{U}(j) e^{i j \theta / h},
$$

where

$$
\widehat{U}(j)=\frac{1}{(2 \pi h)^{1 / 2}} \int_{-h \pi}^{h \pi} U(\theta) e^{-i j \theta / h} d \theta, \quad j \in \mathbb{Z}
$$


Given $u: \mathbb{Z}_{h} \rightarrow \mathbb{R}$, its Fourier transform is a function defined on $[-h \pi, h \pi)$ whose Fourier coefficients are given by the sequence $\left\{u_{j}\right\}_{j \in \mathbb{Z}}$. In other words, if $u \in \ell_{h}^{1}$ then we define

$$
\mathcal{F}_{\mathbb{Z}_{h}} u(\theta)=\sum_{j \in \mathbb{Z}} u_{j} e^{i j \theta / h}, \quad \theta \in[-h \pi, h \pi) .
$$

Then the operator $u \mapsto \mathcal{F}_{\mathbb{Z}_{h}} u$ extends as an isometry from $\ell_{h}^{2}$ into $L_{h}^{2}$, with inverse given by

$$
\mathcal{F}_{\mathbb{Z}_{h}}^{-1} U(j)=\widehat{U}(j) .
$$

Let us then prove (6.2). We can easily check that if $u \in \ell_{h}^{2}$ then

$$
\mathcal{F}_{\mathbb{Z}_{h}}\left(-\Delta_{h} u\right)(\theta)=\left[\frac{4}{h^{2}} \sin ^{2}\left(\frac{\theta}{2 h}\right)\right] \mathcal{F}_{\mathbb{Z}_{h}} u(\theta) .
$$

It is a simple exercise to verify that our semigroup definition 1.3 coincides with the Fourier transform definition

$$
\mathcal{F}_{\mathbb{Z}_{h}}\left[\left(-\Delta_{h}\right)^{s} u\right](\theta)=\left[\frac{4}{h^{2}} \sin ^{2}\left(\frac{\theta}{2 h}\right)\right]^{s} \mathcal{F}_{\mathbb{Z}_{h}} u(\theta),
$$

for $0<s<1$. Then 6.2 follows by noticing that the Fourier multiplier

$$
m_{s}(\theta)=\left[\frac{4}{h^{2}} \sin ^{2}\left(\frac{\theta}{2 h}\right)\right]^{s}, \quad \theta \in \mathbb{T}_{h},
$$

is a bounded function on $[-h \pi, h \pi)$, with

$$
\left\|m_{s}\right\|_{L^{\infty}\left(\mathbb{T}_{h}\right)}=\frac{4^{s}}{h^{2 s}} .
$$

Lemma 6.2. Let $u, v \in \ell_{h}^{2}$. Then, for any $0<s<1$,

$$
\begin{aligned}
\left\langle\left(-\Delta_{h}\right)^{s} u, v\right\rangle_{\ell_{h}^{2}} & =\left\langle\left(-\Delta_{h}\right)^{s / 2} u,\left(-\Delta_{h}\right)^{s / 2} v\right\rangle_{\ell_{h}^{2}} \\
& =\frac{h}{2} \sum_{j \in \mathbb{Z}} \sum_{m \in \mathbb{Z}, m \neq j}\left(u_{j}-u_{m}\right)\left(v_{j}-v_{m}\right) K_{s}^{h}(j-m) .
\end{aligned}
$$

Proof. In view of $(6.2)$ we can use Plancherel's identity and the Fourier transform characterization 6.3 to write

$$
\begin{aligned}
\left\langle\left(-\Delta_{h}\right)^{s / 2} u,\left(-\Delta_{h}\right)^{s / 2} v\right\rangle_{\ell_{h}^{2}} & =\left\langle u,\left(-\Delta_{h}\right)^{s} v\right\rangle_{\ell_{h}^{2}} \\
& =h \sum_{j \in \mathbb{Z}} u_{j}\left(-\Delta_{h}\right)^{s} v_{j} \\
& =h \sum_{j \in \mathbb{Z}} \sum_{m \in \mathbb{Z}, m \neq j} u_{j}\left(v_{j}-v_{m}\right) K_{s}^{h}(j-m) .
\end{aligned}
$$

By interchanging the roles of $j$ and $m$ above and using Fubini's Theorem and the symmetry $K_{s}^{h}(m-j)=K_{s}^{h}(j-m)$, we can also write

$$
\begin{aligned}
\left\langle\left(-\Delta_{h}\right)^{s / 2} u,\left(-\Delta_{h}\right)^{s / 2} v\right\rangle_{\ell_{h}^{2}} & =h \sum_{m \in \mathbb{Z}} \sum_{j \in \mathbb{Z}, j \neq m} u_{m}\left(v_{m}-v_{j}\right) K_{s}^{h}(m-j) \\
& =-h \sum_{j \in \mathbb{Z}} \sum_{m \in \mathbb{Z}, m \neq j} u_{m}\left(v_{j}-v_{m}\right) K_{s}^{h}(j-m) .
\end{aligned}
$$

After adding (6.4) and (6.5) we get the conclusion. 
Remark 6.3. It is clear from (6.4) and the Cauchy-Schwarz inequality that if $u \in \ell_{h}^{2}$ then the following interpolation inequality holds:

$$
\left\|\left(-\Delta_{h}\right)^{s / 2} u\right\|_{\ell_{h}^{2}} \leq\|u\|_{\ell_{h}^{2}}\left\|\left(-\Delta_{h}\right)^{s} u\right\|_{\ell_{h}^{2}} .
$$

The following important result will be proved in Subsection 9.3 .

Theorem 6.4 (Fractional discrete Sobolev and Poincaré inequalities). Let $0<s<1 / 2$. There is a constant $C_{s}>0$ depending only on s such that the fractional discrete Sobolev inequality

$$
\|u\|_{\ell_{h}^{2 /(1-2 s)}} \leq C_{s}\left\|\left(-\Delta_{h}\right)^{s / 2} u\right\|_{\ell_{h}^{2}}=C_{s}\left(\frac{h}{2} \sum_{j \in \mathbb{Z}} \sum_{m \in \mathbb{Z}, m \neq j}\left|u_{j}-u_{m}\right|^{2} K_{s}^{h}(j-m)\right)^{1 / 2},
$$

holds for any function $u: \mathbb{Z}_{h} \rightarrow \mathbb{R}$ with compact support $\operatorname{supp}(u) \subset \mathbb{Z}_{h}$. In particular, we have the fractional discrete Poincaré inequality

$$
\|u\|_{\ell_{h}^{2}} \leq C_{s} h^{s}(\# h \operatorname{supp}(u))^{s}\left\|\left(-\Delta_{h}\right)^{s / 2} u\right\|_{\ell_{h}^{2}},
$$

where, for a set $E \subset \mathbb{Z}_{h}$, the notation $\#_{h} E$ means the number of points in $E$.

Lemma 6.5. Let $0<s<1 / 2$. If we endow the set of functions

$$
H_{0}^{s}\left(B^{h}\right):=\left\{u: \mathbb{Z}_{h} \rightarrow \mathbb{R}: u=0 \text { on } \mathbb{Z}_{h} \backslash B^{h}\right\},
$$

with the inner product

$$
\langle u, v\rangle_{H_{0}^{s}\left(B^{h}\right)}=\left\langle\left(-\Delta_{h}\right)^{s / 2} u,\left(-\Delta_{h}\right)^{s / 2} v\right\rangle_{\ell_{h}^{2}}
$$

then $H_{0}^{s}\left(B^{h}\right)$ is a Hilbert space.

Proof. Clearly $H_{0}^{s}\left(B^{h}\right)$ is a linear space and the bilinear form $\langle u, v\rangle_{H_{0}^{s}\left(B^{h}\right)}$ is symmetric, with $\langle u, u\rangle_{H_{0}^{s}\left(B^{h}\right)} \geq 0$ for all $u \in H_{0}^{s}\left(B^{h}\right)$. Let us check that $\langle u, u\rangle_{H_{0}^{s}\left(B^{h}\right)}=0$ implies $u=0$. Indeed, we have

$$
0 \leq \frac{h}{2} \sum_{j \in \mathbb{Z}} \sum_{m \in \mathbb{Z}, m \neq j}\left|u_{j}-u_{m}\right|^{2} K_{s}^{h}(j-m)=0 .
$$

Since the kernel $K_{s}^{h}\left(j-m\right.$ ) is positive for $j \neq m$ (see (1.7)), we get $u_{j}=u_{m}$ for all $j \neq m$. As $u_{m}$ is zero for all $m$ outside $B^{h}$, we get $u=0$ everywhere. Another way of proving that $u=0$ is by means of the fractional discrete Poincaré inequality (6.6). To show completeness, suppose that $\left(u^{k}\right)_{k \geq 0}$ is a Cauchy sequence in $H_{0}^{s}\left(B^{h}\right)$. Then, by the Poincare inequality (6.6), we see that $\left(u^{k}\right)_{k \geq 0}$ is a Cauchy sequence in $\ell_{h}^{2}$ and so it has a pointwise limit $u \in \ell_{h}^{2}$. Observe that $u=0$ in $\mathbb{Z}_{h} \backslash B^{h}$ and so, in view of $(6.2), u \in H_{0}^{s}\left(B^{h}\right)$. Moreover, again by (6.2), $u^{k} \rightarrow u$ in $H_{0}^{s}\left(B^{h}\right)$, as $k \rightarrow \infty$.

Proof of Theorem 6.1. We say that $u: \mathbb{Z}_{h} \rightarrow \mathbb{R}$ is a weak solution to 6.1 if $u \in H_{0}^{s}\left(B^{h}\right)$ and

$$
\langle u, v\rangle_{H_{0}^{s}\left(B^{h}\right)}=\left\langle\left(-\Delta_{h}\right)^{s / 2} u,\left(-\Delta_{h}\right)^{s / 2} v\right\rangle_{\ell_{h}^{2}}=h \sum_{j \in \mathbb{Z}} f_{j} v_{j}=\langle f, v\rangle_{\ell_{h}^{2}},
$$

for all $v \in H_{0}^{s}\left(B^{h}\right)$. Let us show that $v \mapsto\langle f, v\rangle_{\ell_{h}^{2}}$ is a bounded linear functional in $H_{0}^{s}\left(B^{h}\right)$. By Hölder's inequality (3.3) with $p=p^{\prime}=2$ and the Poincaré inequality (6.6),

$$
\left|h \sum_{j \in \mathbb{Z}} f_{j} v_{j}\right| \leq\|f\|_{\ell_{h}^{2}}\|v\|_{\ell_{h}^{2}} \leq\|f\|_{\ell_{h}^{2}} C_{s} h^{s}\left(\# B_{h} B^{h}\|v\|_{H_{0}^{s}\left(B^{h}\right)} .\right.
$$


Hence the Riesz representation theorem applies and shows that for any given $f$ there is a unique weak solution $u \in H_{0}^{s}\left(B_{h}\right)$. The fact that the first equation in (6.1) holds (that is, that $u$ is a classical solution) follows because Lemma 6.2 shows that $\left\langle\left(-\Delta_{h}\right)^{s} u, v\right\rangle_{\ell_{h}^{2}}=\langle f, v\rangle_{\ell_{h}^{2}}$, for all $v: \mathbb{Z}_{h} \rightarrow \mathbb{R}$ such that $v=0$ in $\mathbb{Z}_{h} \backslash B^{h}$.

\section{The DisCRETE MAXIMUM PRINCIPLE}

To complete the proof of Theorem 1.8 we need the following maximum principle.

Theorem 7.1 (Discrete maximum principle). Let $0<s<1$. Fix an interval $B_{R}^{h} \subset \mathbb{Z}_{h}$, $R>0$. Suppose that $f \in \ell_{h}^{\infty}\left(B_{R}^{h}\right)$ and $g \in \ell_{h}^{\infty}\left(\mathbb{Z}_{h} \backslash B_{R}^{h}\right)$. If $u$ is a solution to

$$
\begin{cases}\left(-\Delta_{h}\right)^{s} u=f, & \text { in } B_{R}^{h} \\ u=g, & \text { in } \mathbb{Z}_{h} \backslash B_{R}^{h}\end{cases}
$$

Then there is a universal constant $C>0$ independent of $s, R$ and $h$ such that

$$
\|u\|_{\ell_{h}^{\infty}\left(B_{R}^{h}\right)} \leq C R^{2 s}\|f\|_{\ell_{h}^{\infty}\left(B_{R}^{h}\right)}+\|g\|_{\ell_{h}^{\infty}\left(\mathbb{Z}_{h} \backslash B_{R}^{h}\right)} .
$$

In particular, uniqueness holds for the Dirichlet problem (7.1).

For the proof we need a barrier which is constructed in Lemma 7.2 and the nonlocal maximum principle stated in Lemma 7.3 (see also [12]).

By the symmetry of the kernel $K_{s}^{h} \frac{(m)}{(n)} m \neq 0$ we can always write

$$
\left(-\Delta_{h}\right)^{s} u_{j}=\frac{1}{2} \sum_{m \neq 0}\left(2 u_{j}-u_{j-m}-u_{j+m}\right) K_{s}^{h}(m) .
$$

Lemma 7.2. Let $0<s<1$ and $R>0$. Define

$$
W(x)= \begin{cases}4 R^{2}-|x|^{2}, & \text { for }|x|<R \\ 0, & \text { for }|x| \geq R\end{cases}
$$

Then the function $w_{j}:=\left(r_{h} W\right)_{j}=W(h j)$ satisfies

$$
\begin{cases}\left(-\Delta_{h}\right)^{s} w \geq M R^{2-2 s}, & \text { in } B_{R}^{h}, \\ w=0, & \text { in } \mathbb{Z}_{h} \backslash B_{R}^{h},\end{cases}
$$

where $M>0$ is a constant independent of $R, s$ and $h$.

Proof. First, for each $h m \in \mathbb{Z}_{h}$, it is not difficult to prove that

$$
2 w_{j}-w_{j-m}-w_{j+m} \geq \min \left\{2 R^{2}, 2|h m|^{2}\right\}, \quad \text { for } h j \in B_{R}^{h},
$$

see, for example, the proof of [12, Lemma 5] for $R=1$. With this and taking into account 7.2 we have, for $h j \in B_{R}^{h}$,

$$
\left(-\Delta_{h}\right)^{s} w_{j} \geq \frac{1}{2} \sum_{m \neq 0} \min \left\{2 R^{2}, 2|h m|^{2}\right\} K_{s}^{h}(m)=R^{2} \sum_{|h m| \geq R} K_{s}^{h}(m)+h^{2} \sum_{\substack{|h m|<R \\ m \neq 0}}|m|^{2} K_{s}^{h}(m) .
$$


Now we use the explicit expression 1.7$)$ and Lemma $9.2(b)$. Then, there exist constants $C_{1}$ and $C_{2}$ independent of $R, s$ and $h$ such that, on one hand,

$$
\begin{aligned}
R^{2} \sum_{|h m| \geq R} K_{s}^{h}(m) & \geq \frac{R^{2}}{h^{2 s}} \sum_{|m| \geq R / h} \frac{4^{s} \Gamma(1 / 2+s)}{2^{1+2 s} \sqrt{\pi}|\Gamma(-s)|} \frac{1}{|m|^{1+2 s}} \\
& \geq C_{1} \frac{\Gamma(1 / 2+s)}{2 \sqrt{\pi}|\Gamma(-s)|} \frac{R^{2}}{h^{2 s}} \int_{|x| \geq R / h} \frac{1}{|x|^{1+2 s}} d x \\
& =C_{1} \frac{\Gamma(1 / 2+s)}{2 s \sqrt{\pi}|\Gamma(-s)|} R^{2-2 s}
\end{aligned}
$$

and, on the other hand,

$$
\begin{aligned}
h^{2} \sum_{\substack{|h m|<R \\
m \neq 0}}|m|^{2} K_{s}^{h}(m) & \geq \frac{h^{2}}{h^{2 s}} \sum_{\substack{|m| \leq R / h \\
m \neq 0}} \frac{4^{s} \Gamma(1 / 2+s)}{2^{1+2 s} \sqrt{\pi}|\Gamma(-s)|} \frac{|m|^{2}}{|m|^{1+2 s}} \\
& \geq C_{2} \frac{\Gamma(1 / 2+s)}{2 \sqrt{\pi}|\Gamma(-s)|} h^{2-2 s} \int_{|x| \leq R / h} \frac{|x|^{2}}{|x|^{1+2 s}} d x \\
& =C_{2} \frac{\Gamma(1 / 2+s)}{(2-2 s) \sqrt{\pi}|\Gamma(-s)|} R^{2-2 s}
\end{aligned}
$$

Altogether,

$$
\left(-\Delta_{h}\right)^{s} w_{j} \geq \min \left\{C_{1}, C_{2}\right\} \frac{\Gamma(1 / 2+s)}{\sqrt{\pi}|\Gamma(-s)|}\left[\frac{1}{2 s}+\frac{1}{2-2 s}\right] R^{2-2 s}>M R^{2-2 s},
$$

where $M>0$ is a constant independent of $R, s$ and $h$, because $\frac{\Gamma(1 / 2+s)}{\sqrt{\pi}|\Gamma(-s)|}\left(\frac{1}{2 s}+\frac{1}{2-2 s}\right)>\frac{1}{4}$.

Lemma 7.3. Let $v: \mathbb{Z}_{h} \rightarrow \mathbb{R}$ be a subsolution to $\left(-\Delta_{h}\right)^{s} v \leq 0$ in an interval $B^{h} \subset \mathbb{Z}_{h}$. Then

$$
\max _{B^{h}} v \leq \sup _{\mathbb{Z}_{h} \backslash B^{h}} v
$$

Similarly, if $v: \mathbb{Z}_{h} \rightarrow \mathbb{R}$ is a supersolution to $\left(-\Delta_{h}\right)^{s} v \geq 0$ in $B^{h} \subset \mathbb{Z}_{h}$ then

$$
\min _{B^{h}} v \geq \inf _{\mathbb{Z}_{h} \backslash B^{h}} v .
$$

Proof. By considering $-v$ in place of $v$, it is enough to prove the result for subsolutions. We argue by contradiction. Suppose that the maximum of $v$ in $B^{h}$, which is attained at a point $h j_{0} \in B^{h}$, is strictly larger than $\sup _{\mathbb{Z}_{h} \backslash B^{h}} v$. Then there exists $h m_{0} \neq h j_{0}$ such that $v_{j_{0}}>v_{m_{0}}$. Hence, by hypothesis and since $v_{j_{0}}-v_{m} \geq 0$ for all $m \neq j_{0}$, we get

$$
0 \geq\left(-\Delta_{h}\right)^{s} v_{j_{0}}=\sum_{m \neq j_{0}}\left(v_{j_{0}}-v_{m}\right) K_{s}^{h}\left(j_{0}-m\right) \geq\left(v_{j_{0}}-v_{m_{0}}\right) K_{s}^{h}\left(j_{0}-m_{0}\right)>0,
$$

which is a contradiction. Then the maximum of $v$ in $B^{h}$ cannot be strictly larger than $\sup _{\mathbb{Z}_{h} \backslash B^{h}} v$.

Proof of Theorem 7.1. Set

$$
v=u-M^{-1} R^{2 s-2}\left\|\left(-\Delta_{h}\right)^{s} u\right\|_{\ell_{h}^{\infty}\left(B_{R}^{h}\right)} w,
$$

where $w$ and $M$ are as in Lemma 7.2. Then, for any $h j \in B_{R}^{h}$,

$$
\left(-\Delta_{h}\right)^{s} v_{j}=\left(-\Delta_{h}\right)^{s} u_{j}-M^{-1} R^{2 s-2}\left\|\left(-\Delta_{h}\right)^{s} u\right\|_{\ell_{h}^{\infty}\left(B_{R}^{h}\right)}\left(-\Delta_{h}\right)^{s} w_{j} \leq 0 .
$$


Thus, by the maximum principle in Lemma 7.3. $\max _{B_{R}^{h}} v \leq \sup _{\mathbb{Z}_{h} \backslash B_{R}^{h}} v$. On the other hand, since $w=0$ on $\mathbb{Z}_{h} \backslash B_{R}^{h}$, we have

$$
\sup _{\mathbb{Z}_{h} \backslash B_{R}^{h}} v=\sup _{\mathbb{Z}_{h} \backslash B_{R}^{h}} u
$$

Thus, as $0 \leq w \leq 4 R^{2}$ on $B_{R}^{h}$,

$$
\begin{aligned}
\max _{B_{R}^{h}} u & \leq \sup _{\mathbb{Z}_{h} \backslash B_{R}^{h}} v+M^{-1} R^{2 s-2}\left\|\left(-\Delta_{h}\right)^{s} u\right\|_{\ell_{h}^{\infty}\left(B_{R}^{h}\right)} \max _{B_{R}^{h}} w \\
& \leq\|u\|_{\ell_{h}^{\infty}\left(\mathbb{Z}_{h} \backslash B_{R}^{h}\right)}+4 M^{-1} R^{2 s}\left\|\left(-\Delta_{h}\right)^{s} u\right\|_{\ell_{h}^{\infty}\left(B_{R}^{h}\right)} \\
& =\|g\|_{\ell_{h}^{\infty}\left(\mathbb{Z}_{h} \backslash B_{R}^{h}\right)}+4 M^{-1} R^{2 s}\|f\|_{\ell_{h}^{\infty}\left(B_{R}^{h}\right)} .
\end{aligned}
$$

Similarly, it can be proved that

$$
\min _{B_{R}^{h}} u \geq-\|u\|_{\ell_{h}^{\infty}\left(\mathbb{Z}_{h} \backslash B_{R}^{h}\right)}-4 M^{-1} R^{2 s}\left\|\left(-\Delta_{h}\right)^{s} u\right\|_{\ell_{h}^{\infty}\left(B_{R}^{h}\right)} .
$$

The proof is complete.

\section{Proof of Theorem 1.8}

Let $v=r_{h} U-u$. Then

By Theorem 7.1

$$
\begin{cases}\left(-\Delta_{h}\right)^{s} v=\left(-\Delta_{h}\right)^{s} r_{h} U-f, & \text { in } B_{R}^{h} \\ v=r_{h} U, & \text { in } \mathbb{Z}_{h} \backslash B_{R}^{h}\end{cases}
$$

$$
\left\|r_{h} U-u\right\|_{\ell_{h}^{\infty}\left(B_{R}^{h}\right)} \leq C R^{2 s}\left\|\left(-\Delta_{h}\right)^{s}\left(r_{h} U\right)-f\right\|_{\ell_{h}^{\infty}\left(B_{R}^{h}\right)}+\left\|r_{h} U\right\|_{\ell_{h}^{\infty}\left(\mathbb{Z}_{h} \backslash B_{R}^{h}\right)} .
$$

Since $f=r_{h}\left((-\Delta)^{s} U\right)$ and $U \in C^{0, \alpha+2 s}$, Theorem 1.7 $(i)$ implies that the first term above is bounded by $C R^{2 s}[U]_{C^{0, \alpha+2 s}} h^{\alpha}$, where $C$ is independent of $R$ and $h$. For the second term, we clearly have $\left\|r_{h} U\right\|_{\ell^{\infty}\left(\mathbb{Z}_{h} \backslash B_{R}^{h}\right)} \leq\|U\|_{L^{\infty}\left(\mathbb{R} \backslash B_{R}\right)}$. From the results in Theorem 9.9, we have

$$
[U]_{C^{0, \alpha+2 s}} \leq C_{\alpha, s, R_{0}}\|F\|_{C^{0, \alpha}},
$$

see 9.20 . Moreover, for any $x \in \mathbb{R} \backslash B_{R}$ with $R>2 R_{0}$, we have (see 9.20)

$$
|U(x)| \leq A_{-s} \int_{B_{R_{0}}} \frac{|F(y)|}{|x-y|^{1-2 s}} d y \leq A_{-s} \frac{\|F\|_{L^{\infty}}}{R_{0}^{1-2 s}} \leq C \frac{\|F\|_{L^{\infty}}}{R^{1-2 s}},
$$

where $C>0$ depends only on $s$. Hence, with our choice of $R$,

$$
\left\|r_{h} U-u\right\|_{\ell^{\infty}\left(B_{R}^{h}\right)} \leq C_{\alpha, s, R_{0}}\|F\|_{C^{0, \alpha}} R^{2 s}\left(h^{\alpha}+R^{-1}\right) \leq C_{\alpha, s, R_{0}}\|F\|_{C^{0, \alpha}} R^{2 s} h^{\alpha},
$$

where $C_{\alpha, s, R_{0}}>0$ is independent of $R$ and $h$.

9. Technical lemmas, Bessel functions, the continuous Poisson problem and The Fractional Discrete Sobolev and Poincaré inequalities

9.1. Some technical lemmas. Lemmas in this subsection are needed in the proof of Theorem 1.7. They are also useful to get estimates for the kernels of the fractional discrete Laplacian in Theorem 1.1 and for the fractional integral kernel in Theorem 1.3 .

Lemma 9.1. Let $\lambda>0$. Let $a, b$ be real numbers such that $0 \leq a<b<\infty$. Then

$$
\min \{\lambda, 1\} \leq \frac{b^{\lambda}-a^{\lambda}}{b^{\lambda-1}(b-a)} \leq \max \{\lambda, 1\}
$$


Proof. Let us first suppose that $\lambda \geq 1$. Then, as $0 \leq a<b<\infty$, we have

$$
0 \leq a / b<1 \Rightarrow 0 \leq(a / b)^{\lambda} \leq a / b<1 \Rightarrow \frac{b^{\lambda}-a^{\lambda}}{b^{\lambda-1}(b-a)}=\frac{1-(a / b)^{\lambda}}{1-a / b} \geq 1 .
$$

On the other hand, by applying the mean value theorem to the function $t \mapsto t^{\lambda}$, we get

$$
\frac{b^{\lambda}-a^{\lambda}}{b^{\lambda-1}(b-a)}=\frac{1-(a / b)^{\lambda}}{1-a / b}=\lambda x^{\lambda-1} \leq \lambda,
$$

for certain $x \in(a / b, 1)$. In the case $0<\lambda<1$, the proof is analogous.

Lemma 9.2. Let $0<s<1, t \in \mathbb{R}$, and $m \in \mathbb{Z}, m \neq 0$.

(a) We have

$$
\left|\frac{\Gamma(|m|-s)}{\Gamma(|m|+1+s)}-\frac{1}{|m|^{1+2 s}}\right| \leq \frac{C_{s}}{|m|^{2+2 s}}
$$

In the case when $0<s<1 / 2$, we have

$$
\left|\frac{\Gamma(|m|+s)}{\Gamma(|m|+1-s)}-\frac{1}{|m|^{1-2 s}}\right| \leq \frac{C_{s}}{|m|^{2-2 s}} .
$$

The constants $C_{s}>0$ above depend only on $s$.

(b) We have the lower bounds

$$
\frac{\Gamma(|m|-s)}{\Gamma(|m|+1+s)} \geq \frac{1}{(2|m|)^{1+2 s}}
$$

and, for $0<s<1 / 2$,

$$
\frac{\Gamma(|m|+s)}{\Gamma(|m|+1-s)} \geq \frac{1}{(2|m|)^{1-2 s}}
$$

Proof. Without loss of generality, $m>0$. We begin with the proof of 9.1 in $(a)$. We write

$$
\left|\frac{\Gamma(m-s)}{\Gamma(m+1+s)}-\frac{1}{m^{1+2 s}}\right| \leq\left|\frac{\Gamma(m-s)}{\Gamma(m+1+s)}-\frac{1}{(m-s)^{1+2 s}}\right|+\left|\frac{1}{(m-s)^{1+2 s}}-\frac{1}{m^{1+2 s}}\right| .
$$

The second term can be easily estimated, just by applying Lemma 9.1 with $\lambda=1+2 s, a=\frac{1}{m}$ and $b=\frac{1}{m-s}$, namely,

$$
\left|\frac{1}{(m-s)^{1+2 s}}-\frac{1}{m^{1+2 s}}\right| \sim \frac{1}{(m-s)^{2 s}}\left(\frac{1}{m-s}-\frac{1}{m}\right) \sim \frac{C_{s}}{m^{2+2 s}},
$$

where the symbol $\sim$ means that constants depend only on $s$. Now we study the first term. By recalling (5.1), for $k \in \mathbb{N}$, we have

$$
\frac{\Gamma(k-s)}{\Gamma(k+n+s)}=\frac{1}{\Gamma(n+2 s)} \int_{0}^{\infty} e^{-(k-s) v}\left(1-e^{-v}\right)^{n+2 s-1} d v .
$$


With this,

$$
\begin{aligned}
\Gamma(1+2 s)\left|\frac{\Gamma(m-s)}{\Gamma(m+1+s)}-\frac{1}{(m-s)^{1+2 s}}\right| & \leq \int_{0}^{\infty} e^{-(m-s) v}\left|v^{2 s}-\left(1-e^{-v}\right)^{2 s}\right| d v \\
& =\int_{0}^{\infty} e^{-(m-s) v} v^{2 s}\left|1-\left(\frac{1-e^{-v}}{v}\right)^{2 s}\right| d v \\
& \sim \int_{0}^{\infty} e^{-(m-s) v} v^{2 s}\left|1-\frac{1-e^{-v}}{v}\right| d v \\
& \leq \frac{1}{2} \int_{0}^{\infty} e^{-(m-s) v} v^{2 s+1} d v \sim \frac{\Gamma(1+2 s)}{2} \frac{1}{m^{2+2 s}}
\end{aligned}
$$

where we applied Lemma 9.1, and in the last inequality we used that $\frac{v^{2}}{2}>v-1+e^{-v}$ for $v \in(0, \infty)$. The proof of $(9.2)$ is analogous, with the restriction $0<s<1 / 2$ coming from Lemma 9.1 .

The proof of the first estimate in (b) follows from (5.1) and an application of the Mean Value Theorem, namely,

$$
\begin{aligned}
\frac{1}{\Gamma(1+2 s)} \int_{0}^{\infty} e^{-(m-s) v}\left(1-e^{-v}\right)^{2 s} d v & \geq \frac{1}{\Gamma(1+2 s)} \int_{0}^{\infty} e^{-(m-s) v} e^{-2 s v} v^{2 s} d v \\
& =\frac{1}{(m+s)^{1+2 s}} \geq \frac{1}{2^{1+2 s} m^{1+2 s}} .
\end{aligned}
$$

In a similar way we can get the second bound in $(b)$ after choosing $z=m, \alpha=s \in(0,1 / 2)$ and $\beta=1-s$ in (5.1).

9.2. Properties of Bessel functions $I_{k}$. We collect in this subsection some properties of modified Bessel functions. Let $I_{k}$ be the modified Bessel function of the first kind and order $k \in \mathbb{Z}$, defined as

$$
I_{k}(t)=i^{-k} J_{k}(i t)=\sum_{m=0}^{\infty} \frac{1}{m ! \Gamma(m+k+1)}\left(\frac{t}{2}\right)^{2 m+k} .
$$

Since $k$ is an integer and $1 / \Gamma(n)$ is taken to be equal zero if $n=0,-1,-2, \ldots$, the function $I_{k}$ is defined in the whole real line. It is verified that

$$
I_{-k}(t)=I_{k}(t),
$$

for each $k \in \mathbb{Z}$. Besides, from $(9.3)$ it is clear that $I_{0}(0)=1$ and $I_{k}(0)=0$ for $k \neq 0$. Also,

$$
I_{k}(t) \geq 0
$$

for every $k \in \mathbb{Z}$ and $t \geq 0$, and

$$
\sum_{k \in \mathbb{Z}} e^{-2 t} I_{k}(2 t)=1
$$

On the other hand, there exist constants $C, c>0$ such that

$$
c t^{k} \leq I_{k}(t) \leq C t^{k}, \quad \text { as } t \rightarrow 0^{+} .
$$

In fact,

$$
I_{k}(t) \sim\left(\frac{t}{2}\right)^{k} \frac{1}{\Gamma(k+1)}, \quad \text { for a fixed } k \neq-1,-2,-3, \ldots \text { and } t \rightarrow 0^{+},
$$

see [19]. It is well known (see [15]) that

$$
I_{k}(t)=C e^{t} t^{-1 / 2}+R_{k}(t),
$$


where

$$
\left|R_{k}(t)\right| \leq C_{k} e^{t} t^{-3 / 2}, \quad \text { as } t \rightarrow \infty .
$$

We also have (see [19]) that, as $\nu \rightarrow \infty$,

$$
I_{\nu}(z) \sim \frac{1}{\sqrt{2 \pi \nu}}\left(\frac{e z}{2 \nu}\right)^{\nu} \sim \frac{z^{\nu}}{2^{\nu} \nu !}
$$

For the following formula see [20, p. 305]. For $\operatorname{Re} c>0,-\operatorname{Re} \nu<\operatorname{Re} \alpha<1 / 2$,

$$
\int_{0}^{\infty} e^{-c t} I_{\nu}(c t) t^{\alpha-1} d t=\frac{(2 c)^{-\alpha}}{\sqrt{\pi}} \frac{\Gamma(1 / 2-\alpha) \Gamma(\alpha+\nu)}{\Gamma(\nu+1-\alpha)} .
$$

9.3. The fractional discrete Sobolev and Poincaré inequalities. The aim of this subsection is to prove Theorem 6.4. The idea is to follow the clever elementary proof of the fractional Sobolev inequality presented by O. Savin and E. Valdinoci in [23], which makes use of their Sobolev embedding for sets proved in [24]. We sketch the main steps.

Along this subsection we let

$$
0<s<1 / 2, \text { so that } 2 /(1-2 s)>2 \text {. }
$$

We start by working on the integers $\mathbb{Z}$, then we will see how to get the result for the mesh $\mathbb{Z}_{h}$, for any $h>0$. If $R>0$ we denote the discrete interval

$$
I_{R}:=\{n \in \mathbb{Z}:-R<n<R\} .
$$

In this way, if $R$ is an integer then the measure of the interval above is

$$
\# I_{R}=2 R-1 \text {, }
$$

where we denote by $\# E$ the number of integers points in the set $E \subset \mathbb{Z}$ (counting measure). As in [23], the following discrete Sobolev embedding for sets (which in fact is valid for any $s>0$ ) is crucial in the proof.

Lemma 9.3 (Discrete analogue of [24, Lemma A.1]). Let $k \in \mathbb{Z}$ be fixed. Let $E \subset \mathbb{Z}$ be $a$ nonempty finite set. There exists a constant $C_{s}>0$ depending only on s such that

$$
\sum_{l \notin E} \frac{1}{|k-l|^{1+2 s}} \geq C_{s}(\# E)^{-2 s} .
$$

Proof. We assume that $k \in E$, otherwise the conclusion is trivially true as the left hand side of the inequality is infinite. By replacing $E$ by $E-k$ we can suppose that $k=0 \in E$. Let $R=\# E$. Then $R$ is a positive integer. If we show that

$$
\sum_{l \notin E} \frac{1}{|l|^{1+2 s}} \geq \sum_{l \notin I_{R}} \frac{1}{|l|^{1+2 s}},
$$

then the conclusion follows. Indeed, we can bound

$$
\sum_{l \notin I_{R}} \frac{1}{|l|^{1+2 s}} \geq \int_{R}^{\infty} \frac{1}{x^{1+2 s}} d x=C_{s}(\# E)^{-2 s} .
$$

For 9.12 , we first observe that we can use (9.11) to get

$$
\begin{aligned}
\#\left(E^{c} \cap I_{R}\right) & =\# I_{R}-\#\left(E \cap I_{R}\right) \\
& =(2 R-1)-\#\left(E \cap I_{R}\right) \\
& \geq \# E-\#\left(E \cap I_{R}\right)=\#\left(E \cap I_{R}^{c}\right) .
\end{aligned}
$$


We estimate now by using this last inequality as follows:

$$
\begin{aligned}
\sum_{l \notin E} \frac{1}{|l|^{1+2 s}} & =\sum_{l \notin E, l \in I_{R}} \frac{1}{|l|^{1+2 s}}+\sum_{l \notin E, l \notin I_{R}} \frac{1}{|l|^{1+2 s}} \\
& \geq \sum_{l \notin E, l \in I_{R}} \frac{1}{R^{1+2 s}}+\sum_{l \notin E, l \notin I_{R}} \frac{1}{|l|^{1+2 s}} \\
& =\frac{\#\left(E^{c} \cap I_{R}\right)}{R^{1+2 s}}+\sum_{l \notin E, l \notin I_{R}} \frac{1}{|l|^{1+2 s}} \\
& \geq \frac{\#\left(E \cap I_{R}^{c}\right)}{R^{1+2 s}}+\sum_{l \notin E, l \notin I_{R}} \frac{1}{|l|^{1+2 s}} \\
& \geq \sum_{l \in E, l \notin I_{R}} \frac{1}{|l|^{1+2 s}}+\sum_{l \notin E, l \notin I_{R}} \frac{1}{|l|^{1+2 s}}=\sum_{l \notin I_{R}} \frac{1}{|l|^{1+2 s}}
\end{aligned}
$$

and 9.12 is proved.

Lemma 9.4 (See [23, Lemma 5] with $T=2^{2}>1$ and $n=1$ ). Let $N \in \mathbb{Z}$ and let $a_{j}$ be $a$ bounded, nonnegative, decreasing sequence with $a_{j}=0$ for all $j \geq N$. There is a constant $C_{s}>0$ depending only on $s$ such that

$$
\sum_{j \in \mathbb{Z}} 2^{2 k} a_{j}^{1-2 s} \leq C_{s} \sum_{j \in \mathbb{Z}, a_{j} \neq 0} 2^{2 j} a_{j+1} a_{j}^{-2 s}
$$

Lemma 9.5 (Discrete analogue of [23, Lemma 6]). Let $f: \mathbb{Z} \rightarrow \mathbb{R}$ be a sequence with compact support. Define

$$
a_{j}:=\#\left\{k \in \mathbb{Z}:\left|f_{k}\right|>2^{j}\right\} .
$$

Then there is a constant $C_{s}>0$ depending only on $s$ such that

$$
\sum_{j \in \mathbb{Z}, a_{j} \neq 0} 2^{2 j} a_{j+1} a_{j}^{-2 s} \leq C_{s} \sum_{j \in \mathbb{Z}} \sum_{m \in \mathbb{Z}, m \neq j} \frac{\left|f_{j}-f_{m}\right|^{2}}{|j-m|^{1+2 s}} .
$$

Using Lemmas 9.4 and 9.5 we can prove the following result.

Theorem 9.6 (Discrete analogue of the fractional Sobolev inequality on $\mathbb{Z}$ ). There is a constant $C_{s}>0$ depending only on $s$ such that for any sequence $f: \mathbb{Z} \rightarrow \mathbb{R}$ with compact support,

$$
\left(\sum_{j \in \mathbb{Z}}\left|f_{j}\right|^{2 /(1-2 s)}\right)^{(1-2 s) / 2} \leq C_{s}\left(\sum_{j \in \mathbb{Z}} \sum_{m \in \mathbb{Z}, m \neq j} \frac{\left|f_{j}-f_{m}\right|^{2}}{|j-m|^{1+2 s}}\right)^{1 / 2} .
$$

Proof. It is easy to see that the right hand side of (9.14) is finite (even for the more general case $f \in \ell^{2}$, see $(6.2)$, Lemma 6.2 and $(1.8)$ ). For any $j \in \mathbb{Z}$ we let $A_{j}=\left\{k \in \mathbb{Z}:\left|f_{k}\right|>2^{j}\right\}$. Notice that

$$
A_{j} \supset A_{j+1}, \quad \text { and } \bigcup_{j \in \mathbb{Z}} A_{j}=\mathbb{Z}
$$


Let $a_{j}=\# A_{j}$ as in 9.13 . We can write

$$
\begin{aligned}
\sum_{j \in \mathbb{Z}}\left|f_{j}\right|^{2 /(1-2 s)} & =\sum_{j \in \mathbb{Z}} \sum_{k \in A_{j} \backslash A_{j+1}}\left|f_{k}\right|^{2 /(1-2 s)} \\
& \leq \sum_{j \in \mathbb{Z}}\left(2^{j+1}\right)^{2 /(1-2 s)} \#\left(A_{j} \backslash A_{j+1}\right) \\
& \leq \sum_{j \in \mathbb{Z}} 2^{2(j+1) /(1-2 s)} a_{j} .
\end{aligned}
$$

As $1-2 s<1$, the function $\varphi(t)=t^{1-2 s}, t \geq 0$, is concave in $[0, \infty)$ and satisfies $\varphi(0)=0$. Hence $\varphi$ is subadditive. Using this and the estimate we just performed above, we get

$$
\left(\sum_{j \in \mathbb{Z}}\left|f_{j}\right|^{2 /(1-2 s)}\right)^{1-2 s} \leq 4 \sum_{j \in \mathbb{Z}} 2^{2 j} a_{j}^{1-2 s} .
$$

Next we verify that the sequence $a_{j}$ satisfies the hypotheses of Lemma 9.4. As $f$ has compact support, we have $A_{j} \subseteq \operatorname{supp}(f)$, for all $j \in \mathbb{Z}$. Then $0 \leq a_{j} \leq \# \operatorname{supp}(f)$, so the sequence $a_{j}$ is uniformly bounded in $j \in \mathbb{Z}$ and each $a_{j}$ is nonnegative. Using (9.15) it follows that $a_{j}$ is decreasing. Finally, as $f$, being a compactly supported sequence of real numbers, is bounded, there is an $N>0$ such that $\left|f_{k}\right|<2^{N}$ for all $k \in \mathbb{Z}$. Hence $A_{j}$ is empty for all $j \geq N$, which gives that $a_{j}=0$ for all $j \geq N$. Thus we can apply Lemma 9.4 to the sequence $a_{j}$. From (9.16), by Lemma 9.4 and Lemma 9.5 , we clearly see that 9.14 follows.

We are left to prove Lemma 9.5, for which we follow [23].

Proof of Lemma 9.5. As ||$f_{j}|-| f_{m}|| \leq\left|f_{j}-f_{m}\right|$, we can assume, by possibly replacing $f$ by $|f|$, that $f \geq 0$. For any $j \in \mathbb{Z}$, let us define

$$
D_{j}=A_{j} \backslash A_{j+1}=\left\{m \in \mathbb{Z}: 2^{j}<f_{m} \leq 2^{j+1}\right\}, \quad \text { and } \quad d_{j}=\# D_{j} .
$$

As $f$ is bounded with compact support, both $a_{j}$ and $d_{j}$ become zero for $j$ large enough. Define the convergent series

$$
S=\sum_{j \in \mathbb{Z}, a_{j-1} \neq 0} 2^{2 j} a_{j-1}^{-2 s} d_{j}
$$

With this notation we have (see the computation for [23, eq. (32)]):

$$
\sum_{j \in \mathbb{Z}, a_{j-1} \neq 0} \sum_{\ell \in \mathbb{Z}, \ell \geq j+1} 2^{2 j} a_{j-1}^{-2 s} d_{\ell} \leq S .
$$

Let $j \in \mathbb{Z}$ and $k \in D_{j}$. Take any $m \leq j-2$ and any $l \in D_{m}$. Then

$$
\left|f_{k}-f_{l}\right| \geq 2^{j}-2^{m+1} \geq 2^{j}-2^{j-1}=2^{j-1}
$$

from which, by Lemma 9.3 and the facts that $\cup_{m \leq j-2} D_{m}=A_{j-1}^{c}$ (disjoint union) and $a_{j-1}=\# A_{j-1}$, we deduce

$$
\begin{aligned}
\sum_{m \in \mathbb{Z}, m \leq j-2} \sum_{l \in D_{m}} \frac{\left|f_{k}-f_{l}\right|^{2}}{|k-l|^{1+2 s}} & \geq 2^{2(j-1)} \sum_{m \in \mathbb{Z}, m \leq j-2} \sum_{l \in D_{m}} \frac{1}{|k-l|^{1+2 s}} \\
& =2^{2(j-1)} \sum_{l \notin A_{j-1}} \frac{1}{|k-l|^{1+2 s}} \\
& \geq C_{s} 2^{2 j} a_{j-1}^{-2 s}
\end{aligned}
$$


Now we sum the inequality above among all $l \in D_{m}$ and use that

$$
d_{j}=a_{j}-\sum_{\ell \geq j+1} d_{\ell}
$$

to get that, for every $j \in \mathbb{Z}$,

$$
\begin{aligned}
\sum_{m \in \mathbb{Z}, m \leq j-2} \sum_{l \in D_{m}} \sum_{k \in D_{j}} \frac{\left|f_{k}-f_{l}\right|^{2}}{|k-l|^{1+2 s}} & \geq C_{0} 2^{2 j} a_{j-1}^{-2 s} d_{j} \\
& =C_{0}\left[2^{2 j} a_{j-1}^{-2 s} a_{j}-\sum_{\ell \in \mathbb{Z}, \ell \geq j+1} 2^{2 j} a_{j-1}^{-2 s} d_{\ell}\right] .
\end{aligned}
$$

We sum for all $j \in \mathbb{Z}$ such that $a_{j-1} \neq 0$ in inequality (9.17) to get

$$
\sum_{j \in \mathbb{Z}, a_{j-1} \neq 0} \sum_{m \in \mathbb{Z}, m \leq j-2} \sum_{l \in D_{m}} \sum_{k \in D_{j}} \frac{\left|f_{k}-f_{l}\right|^{2}}{|k-l|^{1+2 s}} \geq C_{0} \sum_{j \in \mathbb{Z}, a_{j-1} \neq 0} 2^{2 j} a_{j-1}^{-2 s} d_{j}=C_{0} S .
$$

Therefore, as in [23, p. 2685], we reach the analogue of [23, eq. (36)]:

$$
2 \sum_{j \in \mathbb{Z}, a_{j-1} \neq 0} \sum_{m \in \mathbb{Z}, m \leq j-2} \sum_{l \in D_{m}} \sum_{k \in D_{j}} \frac{\left|f_{k}-f_{l}\right|^{2}}{|k-l|^{1+2 s}} \geq C_{0} \sum_{j \in \mathbb{Z}, a_{j-1} \neq 0} 2^{2 j} a_{j-1}^{-2 s} a_{j} .
$$

Finally, by symmetry, we can write

$$
\begin{aligned}
\sum_{j \in \mathbb{Z}} \sum_{m \in \mathbb{Z}, m \neq j} \frac{\left|f_{j}-f_{m}\right|^{2}}{|j-m|^{1+2 s}} & =2 \sum_{j, m \in \mathbb{Z}, m<j} \frac{\left|f_{j}-f_{m}\right|^{2}}{|j-m|^{1+2 s}} \\
& =2 \sum_{j, m \in \mathbb{Z}, m<j} \sum_{k \in D_{j}} \sum_{l \in D_{m}} \frac{\left|f_{k}-f_{l}\right|^{2}}{|k-l|^{1+2 s}} \\
& \geq 2 \sum_{j \in \mathbb{Z}, a_{j-1} \neq 0} \sum_{m \in \mathbb{Z}, m \leq j-2} \sum_{k \in D_{j}} \sum_{l \in D_{m}} \frac{\left|f_{k}-f_{l}\right|^{2}}{|k-l|^{1+2 s}},
\end{aligned}
$$

and from (9.18), the conclusion of Lemma 9.5 follows with $C_{s}=1 / C_{0}$.

Corollary 9.7 (Discrete analogue of Sobolev inequality on $\mathbb{Z}_{h}$ ). Let $u: \mathbb{Z}_{h} \rightarrow \mathbb{R}$ be a function with compact support. Then there is a constant $C_{s}>0$ depending only on s such that

$$
\|u\|_{\ell_{h}^{2 /(1-2 s)}} \leq C_{s}\left(h^{2} \sum_{j \in \mathbb{Z}} \sum_{m \in \mathbb{Z}, m \neq j} \frac{\left|u_{j}-u_{m}\right|^{2}}{|h j-h m|^{1+2 s}}\right)^{1 / 2} .
$$

Proof. Given the function $u$ on $\mathbb{Z}_{h}$ we can define a new function (sequence) $f$ on $\mathbb{Z}_{1}=\mathbb{Z}$ through $f_{j}:=u(h j)=u_{j}$, for $j \in \mathbb{Z}$. With this notation, for any $1 \leq p \leq \infty,\|u\|_{\ell_{h}^{p}}=$ $h^{1 / p}\|f\|_{\ell^{p}}$. Then $f$ is a sequence with compact support, so we can apply (9.14) to it and get

$$
\left(\sum_{j \in \mathbb{Z}}\left|u_{j}\right|^{2 /(1-2 s)}\right)^{(1-2 s) / 2} \leq C_{s}\left(\sum_{j \in \mathbb{Z}} \sum_{m \in \mathbb{Z}, m \neq j} \frac{\left|u_{j}-u_{m}\right|^{2}}{|j-m|^{1+2 s}}\right)^{1 / 2} .
$$

Now we multiply both sides by $h^{(1-2 s) / 2}$ and $(9.19)$ follows. 
Remark 9.8. Observe that the factor $h^{2}$ appearing in the right hand side of $(9.19)$ is the correct one since that expression is nothing but the $\ell^{2}\left(\mathbb{Z}_{h} \times \mathbb{Z}_{h}\right)$ norm of the two-variables function $v=v(h j, h m): \mathbb{Z}_{h} \times \mathbb{Z}_{h} \backslash\{(h j, h m): j=m\} \rightarrow \mathbb{R}$ given by

$$
v(h j, h m)=\frac{\left|u_{j}-u_{m}\right|^{2}}{|h j-h m|^{1+2 s}} .
$$

Proof of Theorem 6.4. By applying (1.8) and Corollary 9.7,

$$
\begin{aligned}
\frac{h}{2} \sum_{j \in \mathbb{Z}} \sum_{m \in \mathbb{Z}, m \neq j}\left|u_{j}-u_{m}\right|^{2} K_{s}^{h}(j-m) & \geq C_{s} h^{2} \sum_{j \in \mathbb{Z}} \sum_{m \in \mathbb{Z}, m \neq j} \frac{\left|u_{j}-u_{m}\right|^{2}}{|h j-h m|^{1+2 s}} \\
& \geq C_{s}\|u\|_{\ell_{h}^{2 /(1-2 s)} .}^{2}
\end{aligned}
$$

and the Sobolev inequality follows. To prove the Poincaré inequality, notice that, by Hölder's inequality 3.3 with $p=1 /(1-2 s)>1$ and $p^{\prime}=1 /(2 s)$,

$$
\begin{aligned}
\|u\|_{\ell_{h}^{2}}^{2} & =h \sum_{h j \in \operatorname{supp}(u)}\left|u_{j}\right|^{2}=\left\|\chi_{\operatorname{supp}(u)} \cdot u^{2}\right\|_{\ell_{h}^{1}} \\
& \leq\left\|\chi_{\operatorname{supp}(u)}\right\|_{\ell_{h}^{1 /(2 s)}}\left\|u^{2}\right\|_{\ell_{h}^{1 /(1-2 s)}} \\
& =h^{2 s}\left(\#{ }_{h} \operatorname{supp}(u)\right)^{2 s}\left(h \sum_{j \in \mathbb{Z}}\left|u_{j}\right|^{2 /(1-2 s)}\right)^{1-2 s} \\
& =h^{2 s}(\# h \operatorname{supp}(u))^{2 s}\|u\|_{\ell_{h}^{2 /(1-2 s)}}^{2} .
\end{aligned}
$$

Then we apply the Sobolev inequality.

9.4. The continuous Poisson problem. In this subsection we prove the following result, which we believe it belongs to the folklore. We present here a more or less self contained proof. From now on we denote the Fourier transform of $G \in L^{1}(\mathbb{R})$ by

$$
\widehat{G}(\xi)=\frac{1}{(2 \pi)^{1 / 2}} \int_{\mathbb{R}} G(x) e^{-i \xi x} d x, \quad \xi \in \mathbb{R} .
$$

Theorem 9.9. Let $F$ be a function in $C^{0, \alpha}$, for some $0<\alpha<1$, having compact support on $\mathbb{R}$. Take $0<s<1$ such that $0<\alpha+2 s<1$. Then the function $U$ defined by

$$
U(x):=(-\Delta)^{-s} F(x)=A_{-s} \int_{\operatorname{supp}(F)} \frac{F(y)}{|x-y|^{1-2 s}} d y,
$$

for $x \in \mathbb{R}$, where $\operatorname{supp}(F)$ denotes the support of $F$ and $A_{-s}>0$ is as in $(1.16)$, is the unique classical solution to the fractional Poisson problem (1.2) such that $|U(x)| \rightarrow 0$ as $|x| \rightarrow \infty$. Moreover, $U \in C^{0, \alpha+2 s}$ and there is a constant $C>0$ depending only on $s, \alpha$ and the measure of $\operatorname{supp}(F)$, such that

$$
\|U\|_{C^{0, \alpha+2 s}} \leq C\|F\|_{C^{0, \alpha}} .
$$

Proof. Without loss of generality we may assume that $F=0$ outside an interval $\left(-R_{0}, R_{0}\right)$, for some $R_{0}>0$. Then we can write

$$
U(x)=A_{-s} \int_{-R_{0}}^{R_{0}} \frac{F(y)}{|x-y|^{1-2 s}} d y .
$$

It is clear that $U$ is well defined because $|y|^{-1+2 s}$ is a locally integrable function in $\mathbb{R}$ and $F$ is bounded. Next we prove a series of properties about $U$ that will complete the proof. 
(1) $U$ is bounded on $\mathbb{R}$ and $|U(x)| \rightarrow 0$ as $|x| \rightarrow \infty$. To see this we first do a computation. Let $r>0$. It is easy to see that the positive function

$$
N_{s, r}(x):=\int_{-r}^{r}|x-y|^{-1+2 s} d y, \quad x \in \mathbb{R},
$$

is Hölder continuous of order $0<2 s<1$ on $\mathbb{R}$ and smooth in $\mathbb{R} \backslash\{-r, r\}$. In particular,

$$
\frac{d}{d x} N_{s, r}(x)=(x+r)^{2 s-1}-(r-x)^{2 s-1}, \quad \text { for any }-r<x<r .
$$

Now, if $|y|<r$ and $|x|>2 r$ then $|x-y|^{-1+2 s} \leq(|x|-r)^{-1+2 s}$. Hence,

$$
\left|N_{s, r}(x)\right| \leq \frac{2 r}{(|x|-r)^{1-2 s}}, \quad \text { for any }|x|>2 r,
$$

which shows that $\left|N_{s, r}(x)\right| \rightarrow 0$ as $|x| \rightarrow \infty$. Thus $N_{s, r} \in C^{0,2 s}$. For our claim (1) we just observe that

$$
|U(x)| \leq A_{-s}\|F\|_{L^{\infty}} N_{s, R_{0}}(x), \quad \text { for any } x \in \mathbb{R} .
$$

(2) Let $\varphi \in C^{\infty}(\mathbb{R})$ such that $\left(1+|x|^{1+2 s}\right) D^{k} \varphi \in L^{\infty}(\mathbb{R})$, for all $k \geq 0$ (that is, $\varphi \in \mathcal{S}_{s}$, see the notation in $[25$, p. 73]). Then

$$
\int_{\mathbb{R}} U(x) \varphi(x) d x=\int_{\mathbb{R}}|\xi|^{-2 s} \widehat{F}(\xi) \widehat{\varphi}(\xi) d \xi .
$$

It is easy to check that both integrals in (9.23) are absolutely convergent. We start by proving the following identity:

$$
A_{-s} \int_{\mathbb{R}} \frac{\varphi(y)}{|y|^{1-2 s}} d y=\frac{1}{(2 \pi)^{1 / 2}} \int_{\mathbb{R}}|\xi|^{-2 s} \overline{\widehat{\varphi}(\xi)} d \xi,
$$

where $\bar{z}$ denotes the complex conjugate of $z \in \mathbb{C}$. It is readily seen that both integrals above are absolutely convergent. By Plancherel's identity and Fubini's Theorem,

$$
\begin{aligned}
\frac{1}{(2 \pi)^{1 / 2}} \int_{\mathbb{R}}|\xi|^{-2 s} \overline{\widehat{\varphi}(\xi)} d \xi & =\frac{1}{\Gamma(s)(2 \pi)^{1 / 2}} \int_{0}^{\infty} \int_{\mathbb{R}} e^{-t|\xi|^{2}} \overline{\hat{\varphi}(\xi)} d \xi \frac{d t}{t^{1-s}} \\
& =\frac{1}{\Gamma(s)} \int_{0}^{\infty} \int_{\mathbb{R}} \frac{e^{-|y|^{2} /(4 t)}}{(4 \pi t)^{1 / 2}} \varphi(y) d y \frac{d t}{t^{1-s}} \\
& =\int_{\mathbb{R}}\left[\frac{1}{\Gamma(s)} \int_{0}^{\infty} \frac{e^{-|y|^{2} /(4 t)}}{(4 \pi t)^{1 / 2}} \frac{d t}{t^{1-s}}\right] \varphi(y) d y \\
& =A_{-s} \int_{\mathbb{R}} \frac{\varphi(y)}{|y|^{1-2 s}} d y .
\end{aligned}
$$

Next, for any fixed $x \in \mathbb{R}$, by the properties of the Fourier transform,

$$
A_{-s} \int_{\mathbb{R}} \frac{\varphi(x-y)}{|y|^{1-2 s}} d y=\int_{\mathbb{R}}|\xi|^{-2 s} \overline{\widehat{\varphi}(-\xi)} e^{i x \xi} d \xi .
$$

By multiplying both sides above by $F(x)$ and integrating in $d x$ we get

$$
A_{-s} \int_{\mathbb{R}} \int_{\mathbb{R}} \frac{F(x) \varphi(x-y)}{|y|^{1-2 s}} d x d y=\int_{\mathbb{R}} \int_{\mathbb{R}}|\xi|^{-2 s} \overline{\widehat{\varphi}(-\xi)} F(x) e^{i x \xi} d x d \xi,
$$

which gives

$$
\int_{\mathbb{R}} U(x) \varphi(x) d x=A_{-s} \int_{\mathbb{R}} \varphi(x) \int_{\mathbb{R}} \frac{F(x-y)}{|y|^{1-2 s}} d y d x=\int_{\mathbb{R}}|\xi|^{-2 s} \widehat{F}(-\xi) \overline{\hat{\varphi}(-\xi)} d \xi .
$$


(3) We have $(-\Delta)^{s} U=F$ in the sense of distributions in $\mathcal{S}_{s}^{\prime}$. As $U$ is bounded, we have that $U \in L_{s}(\mathbb{R})$ (see [25] for the notation), namely,

$$
\int_{\mathbb{R}} \frac{|U(x)|}{1+|x|^{1+2 s}}<\infty .
$$

Then $(-\Delta)^{s} U$ can be defined in the distributional sense: for any function $\psi$ in the Schwartz class $\mathcal{S}$, we have $\left\langle(-\Delta)^{s} U, \psi\right\rangle:=\left\langle U,(-\Delta)^{s} \psi\right\rangle$, see [25, p. 73] for details. The fractional Laplacian of $\psi \in \mathcal{S}$ is defined with the Fourier transform as

$$
\widehat{(-\Delta)^{s}} \psi(\xi)=|\xi|^{2 s} \widehat{\psi}(\xi)
$$

Using the semigroup language and the Fourier transform as in [28, 29] we get

$$
(-\Delta)^{s} \psi(x)=A_{s} \int_{\mathbb{R}} \frac{\psi(x)-\psi(y)}{|x-y|^{1+2 s}} d y,
$$

where $A_{s}$ is as in $(1.9)$. We have that $\varphi:=(-\Delta)^{s} \psi \in \mathcal{S}_{s}$, namely, $\varphi$ is a $C^{\infty}$ function such that $\left(1+|x|^{1+2 s}\right) D^{k} \varphi$ is bounded, for any $k \geq 0$. The latter is claimed in [25, p. 73], but we show it here for $k=0$ (the proof for $k \geq 1$ is the same as the derivatives and the fractional Laplacian commute) because we will need the computation at a later stage. Let us see that

$$
\int_{\mathbb{R}} \frac{|\psi(x)-\psi(y)|}{|x-y|^{1+2 s}} d y \leq C_{s, \psi} \frac{1}{|x|^{1+2 s}}, \quad \text { for all }|x|>1 .
$$

Let $|x|>1$ and $y \in \mathbb{R}$. Suppose that $|x-y|<|x| / 2$. Then, for any intermediate point $\xi$ between $x$ and $y$, we have $|x| \leq|x-\xi|+|\xi| \leq|x-y|+|\xi| \leq|x| / 2+|\xi|$, which gives $|x| \leq 2|\xi|$. As a consequence, by the Mean Value Theorem and using that $\psi \in \mathcal{S}$,

$$
\begin{aligned}
|\psi(x)-\psi(y)| & =(1+|\xi|)^{3}\left|\psi^{\prime}(\xi)\right| \frac{|x-y|}{(1+|\xi|)^{3}} \\
& \leq C_{\psi} \frac{|x-y|}{(1+|x|)^{3}} \leq C_{\psi} \frac{|x-y|}{|x|^{3}} .
\end{aligned}
$$

From here,

$$
\begin{aligned}
\int_{|x-y|<|x| / 2} \frac{|\psi(x)-\psi(y)|}{|x-y|^{1+2 s}} d y & \leq \frac{C_{\psi}}{|x|^{3}} \int_{|x-y|<|x| / 2} \frac{|x-y|}{|x-y|^{1+2 s}} d y \\
& =\frac{C_{s, \psi}}{|x|^{2+2 s}} \leq \frac{C_{s, \psi}}{|x|^{1+2 s}}
\end{aligned}
$$

On the other hand,

$$
\begin{aligned}
\int_{|x-y| \geq|x| / 2} \frac{|\psi(x)-\psi(y)|}{|x-y|^{1+2 s}} d y & \leq C_{s}\left(\frac{|\psi(x)|}{|x|^{2 s}}+\frac{\|\psi\|_{L^{1}(\mathbb{R})}}{|x|^{1+2 s}}\right) \\
& =\frac{C_{s}}{|x|^{1+2 s}}\left(|x \psi(x)|+\|\psi\|_{L^{1}(\mathbb{R})}\right) \leq \frac{C_{s, \psi}}{|x|^{1+2 s}} .
\end{aligned}
$$


Thus 9.24 is proved. Let us finish then the proof of our claim (3). Using that $U \in L_{s}(\mathbb{R})$, (9.23) and Plancherel's identity, for any $\psi \in \mathcal{S}$,

$$
\begin{aligned}
& \left\langle(-\Delta)^{s} U, \psi\right\rangle=\left\langle U,(-\Delta)^{s} \psi\right\rangle=\int_{\mathbb{R}} U(x)(-\Delta)^{s} \psi(x) d x \\
& =\int_{\mathbb{R}}|\xi|^{-2 s} \widehat{F}(\xi) \overline{\left(\overline{-\Delta)^{s}} \psi(\xi)\right.} d \xi \\
& =\int_{\mathbb{R}}|\xi|^{-2 s} \widehat{F}(\xi) \overline{|\xi|^{2 s} \widehat{\psi}(\xi)} d \xi \\
& =\int_{\mathbb{R}} \widehat{F}(\xi) \bar{\psi}(\xi) d \xi=\int_{\mathbb{R}} F(x) \psi(x) d x=\langle F, \psi\rangle .
\end{aligned}
$$

(4) $U$ is in $C^{0, \alpha+2 s}$ and 9.20 holds. We showed in (1) that $U$ is bounded. From 9.22,

$$
\|U\|_{L^{\infty}} \leq C_{s, R_{0}}\|F\|_{L^{\infty}} .
$$

Let $x_{1}, x_{2} \in \mathbb{R}$. Suppose that $\left|x_{1}-x_{2}\right| \geq 1$. Then, by using 9.26 ,

$$
\left|U\left(x_{1}\right)-U\left(x_{2}\right)\right| \leq 2\|U\|_{L^{\infty}} \leq C_{s, R_{0}}\|F\|_{L^{\infty}\left|x_{1}-x_{2}\right|^{\alpha+2 s} .}
$$

Assume next that $\left|x_{1}-x_{2}\right|<1$. Let us take $r>0$ sufficiently large so that $r>$ $R_{0}+\left|x_{1}\right|+\left|x_{2}\right|$. As $F=0$ outside the interval $\left(-R_{0}, R_{0}\right)$ and $r>R_{0}$, we can write

$$
\begin{aligned}
U\left(x_{1}\right)-U\left(x_{2}\right)= & A_{-s} \int_{-r}^{r}\left(F(y)-F\left(x_{1}\right)\right)\left(\left|x_{1}-y\right|^{-1+2 s}-\left|x_{2}-y\right|^{-1+2 s}\right) d y \\
& +A_{-s} F\left(x_{1}\right) \int_{-r}^{r}\left(\left|x_{1}-y\right|^{-1+2 s}-\left|x_{2}-y\right|^{-1+2 s}\right) d y \\
= & A_{-s} \int_{-r}^{r}\left(F(y)-F\left(x_{1}\right)\right)\left(\left|x_{1}-y\right|^{-1+2 s}-\left|x_{2}-y\right|^{-1+2 s}\right) d y \\
& +A_{-s} F\left(x_{1}\right)\left(N_{s, r}\left(x_{1}\right)-N_{s, r}\left(x_{2}\right)\right) .
\end{aligned}
$$

Recall the expression for the derivative of the function $N_{s, r}(x)$ for any $-r<x<r$ given in (9.21). In particular, we can use such a formula for any point between $x_{1}$ and $x_{2}$ because we have chosen $r$ large enough so that $-r<x_{1}, x_{2}<r$. By the Mean Value Theorem, for some $\xi$ between $x_{1}$ and $x_{2}$, we have

$$
\begin{aligned}
\left|N_{s, r}\left(x_{1}\right)-N_{s, r}\left(x_{2}\right)\right| & =\left|N_{s, r}^{\prime}(\xi)\right|\left|x_{1}-x_{2}\right| \\
& \leq\left[\frac{1}{(\xi+r)^{1-2 s}}+\frac{1}{(r-\xi)^{1-2 s}}\right]\left|x_{1}-x_{2}\right| \rightarrow 0,
\end{aligned}
$$

as $r \rightarrow \infty$. Therefore, by taking the limit as $r \rightarrow \infty$ in 9.28 , we see that

$$
\left|U\left(x_{1}\right)-U\left(x_{2}\right)\right| \leq A_{-s} \int_{\mathbb{R}}\left|F(y)-F\left(x_{1}\right)\right||| x_{1}-\left.y\right|^{-1+2 s}-\left|x_{2}-y\right|^{-1+2 s} \mid d y .
$$

The last integral is split into

$$
\int_{\mathbb{R}}=\int_{\left|x_{1}-y\right|<2\left|x_{1}-x_{2}\right|}+\int_{\left|x_{1}-y\right| \geq 2\left|x_{1}-x_{2}\right|}=: I+I I .
$$


If $\left|y-x_{1}\right|<2\left|x_{1}-x_{2}\right|$ then $\left|y-x_{2}\right| \leq 4\left|x_{1}-x_{2}\right|$. Hence

$$
\begin{aligned}
I \leq & \int_{\left|x_{1}-y\right|<2\left|x_{1}-x_{2}\right|} \frac{\left|F(y)-F\left(x_{1}\right)\right|}{\left|x_{1}-y\right|^{1-2 s}} d y+\int_{\left|x_{1}-y\right|<2\left|x_{1}-x_{2}\right|} \frac{\left|F(y)-F\left(x_{1}\right)\right|}{\left|x_{2}-y\right|^{1-2 s}} d y \\
\leq & {[F]_{C^{0, \alpha}} \int_{\left|x_{1}-y\right|<2\left|x_{1}-x_{2}\right|} \frac{\left|y-x_{1}\right|^{\alpha}}{\left|x_{1}-y\right|^{1-2 s}} d y } \\
& +C_{\alpha}[F]_{C^{0, \alpha}} \int_{\left|x_{2}-y\right|<4\left|x_{1}-x_{2}\right|} \frac{\left(\left|y-x_{2}\right|^{\alpha}+\left|x_{2}-x_{1}\right|^{\alpha}\right)}{\left|x_{2}-y\right|^{1-2 s}} d y \\
= & C_{\alpha, s}[F]_{C^{0, \alpha}}\left|x_{1}-x_{2}\right|^{\alpha+2 s} .
\end{aligned}
$$

To estimate the second integral, suppose that $\left|x_{1}-y\right| \geq 2\left|x_{1}-x_{2}\right|$. Let $\xi$ be an intermediate point between $x_{1}$ and $x_{2}$. Then $\left|x_{1}-y\right| \leq\left|x_{1}-\xi\right|+|\xi-y| \leq\left|x_{1}-x_{2}\right|+|\xi-y| \leq$ $\frac{1}{2}\left|x_{1}-y\right|+|\xi-y|$. As a consequence, $\left|x_{1}-y\right|<2|\xi-y|$ and so $|\xi-y|^{-2+2 s} \leq C_{s}\left|x_{1}-y\right|^{-2+2 s}$. Using this, the Mean Value Theorem and the fact that $\alpha+2 s<1$, we can estimate

$$
\begin{aligned}
I I & \leq[F]_{C^{0, \alpha}} \int_{\left|x_{1}-y\right| \geq 2\left|x_{1}-x_{2}\right|}\left|y-x_{1}\right|^{\alpha}|| x_{1}-\left.y\right|^{-1+2 s}-\left|x_{2}-y\right|^{-1+2 s} \mid d y \\
& \leq C_{s}[F]_{C^{0, \alpha}}\left|x_{1}-x_{2}\right| \int_{\left|x_{1}-y\right| \geq 2\left|x_{1}-x_{2}\right|}\left|y-x_{1}\right|^{\alpha}\left|x_{1}-y\right|^{-2+2 s} d y \\
& =C_{\alpha, s}[F]_{C^{0, \alpha}}\left|x_{1}-x_{2}\right|^{\alpha+2 s} .
\end{aligned}
$$

These estimates for $I$ and $I I$, together with (9.26) and (9.27), imply (9.20).

(5) $(-\Delta)^{s} U$ is a well defined $C^{0, \alpha}$-function and is given by the pointwise formula

$$
(-\Delta)^{s} U(x)=A_{s} \int_{\mathbb{R}} \frac{U(x)-U(y)}{|x-y|^{1+2 s}} d y, \quad x \in \mathbb{R} .
$$

The pointwise formula follows from the results of [25, p. 73], see also [28]. Observe that the integral above is absolutely convergent and we have the estimate

$$
\begin{aligned}
\int_{\mathbb{R}} \frac{|U(x)-U(y)|}{|x-y|^{1+2 s}} d y \leq & {[U]_{C^{0, \alpha+2 s}} \int_{|x-y|<1} \frac{|x-y|^{\alpha+2 s}}{|x-y|^{1+2 s}} d y } \\
& +2\|U\|_{L^{\infty}} \int_{|x-y| \geq 1} \frac{1}{|x-y|^{1+2 s}} d y \\
\leq & C_{\alpha, s}\|U\|_{C^{0, \alpha+2 s}} .
\end{aligned}
$$

This estimate is valid uniformly in $x \in \mathbb{R}$, hence $(-\Delta)^{s} U$ is bounded. By [25, p. 74], see also [28, we conclude that $(-\Delta)^{s} U$ is in $C^{0, \alpha}(\mathbb{R})$.

(6) We have

$$
(-\Delta)^{s} U(x)=F(x)
$$

in the pointwise sense. We have seen in $(3)$ that $(-\Delta)^{s} U=F$ in the sense of distributions. In particular, by the computation made in (9.25),

$$
\int_{\mathbb{R}} U(x)(-\Delta)^{s} \psi(x) d x=\int_{\mathbb{R}} F(x) \psi(x) d x,
$$

for any $\psi \in \mathcal{S}$. If we show that

$$
\int_{\mathbb{R}} U(x)(-\Delta)^{s} \psi(x) d x=\int_{\mathbb{R}} \psi(x)(-\Delta)^{s} U(x) d x .
$$

then, by using $(9.30)$, we get $(-\Delta)^{s} U(x)=F(x)$, for a.e. $x \in \mathbb{R}$, and, by continuity, $(-\Delta)^{s} U(x)=F(x)$, for every $x \in \mathbb{R}$. So we are left to show (9.31). Since $(-\Delta)^{s} U$ is 
bounded (see (5)) the integral in the right hand side of $(9.31)$ is absolutely convergent. We write the left hand side of 9.31) as

$$
\int_{\mathbb{R}} U(x)(-\Delta)^{s} \psi(x) d x=A_{s} \int_{\mathbb{R}} \int_{\mathbb{R}} U(x) \frac{\psi(x)-\psi(y)}{|x-y|^{1+2 s}} d y d x,
$$

and

$$
\begin{aligned}
\int_{\mathbb{R}} U(y)(-\Delta)^{s} \psi(y) d y & =A_{s} \int_{\mathbb{R}} \int_{\mathbb{R}} U(y) \frac{\psi(y)-\psi(x)}{|y-x|^{1+2 s}} d x d y \\
& =-A_{s} \int_{\mathbb{R}} \int_{\mathbb{R}} U(y) \frac{\psi(x)-\psi(y)}{|x-y|^{1+2 s}} d y d x .
\end{aligned}
$$

In the second identity in 9.33 we applied Fubini's Theorem. To justify it, observe that 9.24 and 9.29 hold for $\psi \in \mathcal{S}$, so

$$
\begin{aligned}
\int_{\mathbb{R}}|U(y)| \int_{\mathbb{R}} \frac{|\psi(y)-\psi(x)|}{|y-x|^{1+2 s}} d x d y & \leq C_{s, \psi}\left(\int_{|y| \leq 1}|U(y)| d y+\int_{|y|>1} \frac{|U(y)|}{|y|^{1+2 s}} d y\right) \\
& \leq C_{s, \psi}\|U\|_{L^{\infty}}<\infty .
\end{aligned}
$$

By adding 9.32 and 9.33 ,

$$
\int_{\mathbb{R}} U(x)(-\Delta)^{s} \psi(x) d x=\frac{A_{s}}{2} \int_{\mathbb{R}} \int_{\mathbb{R}} \frac{(U(x)-U(y))(\psi(x)-\psi(y))}{|x-y|^{1+2 s}} d y d x .
$$

To show that the right hand side of (9.31) is also equal to the double integral above we only need to verify that Fubini's Theorem can be applied in 9.33 with $U$ and $\psi$ interchanged. But this is simpler now because of (9.29):

$$
\int_{\mathbb{R}}|\psi(y)| \int_{\mathbb{R}} \frac{|U(y)-U(x)|}{|y-x|^{1+2 s}} d x d y \leq C_{\alpha, s}\|U\|_{C^{0, \alpha+2 s}} \int_{\mathbb{R}}|\psi(y)| d y<\infty .
$$

Thus (9.31) is proved.

(7) $U$ is the unique classical solution to $(-\Delta)^{s} U=F$ in $\mathbb{R}$ which vanishes at infinity. The previous items show that $U$ is a classical solution vanishing at infinity. Let $V$ be another classical solution vanishing at infinity. Then the difference $W:=U-V$ satisfies

$$
\left\{\begin{array}{l}
(-\Delta)^{s} W=0, \quad \text { in } \mathbb{R}, \\
|W(x)| \rightarrow 0, \quad \text { as }|x| \rightarrow \infty, \\
W \in L^{\infty}(\mathbb{R}) .
\end{array}\right.
$$

By the Liouville Theorem for the fractional Laplacian (see for example [14]), $W$ is a constant and, since it vanishes at infinity, $W=0$.

Remark 9.10. It is worth noticing that in [26, p. 117] identity (9.23) is shown for $\varphi \in \mathcal{S}$ by using spherical harmonics. Instead, our proof is more elementary (and valid for more general functions $\varphi$ ) in the sense we only use the Gamma function and Plancherel's identity.

Acknowledgments. We would like to thank the referee for detailed comments and suggestions that helped us to improve the presentation of the paper. 


\section{REFERENCES}

[1] G. Acosta and J. P. Borthagaray, A fractional Laplace equation: regularity of solutions and finite element approximations, SIAM J. Numer. Anal. 55 (2017), 472-495.

[2] A. Bonito and J. Pasciak, Numerical approximation of fractional powers of elliptic operators, Math. Comp. 84 (2015), 2083-2110.

[3] L. A. Caffarelli, Some nonlinear problems involving non-local diffusions, ICIAM 07-6th International Congress on Industrial and Applied Mathematics, 43-56, Eur. Math. Soc., Zürich, 2009.

[4] L. A. Caffarelli and L. Silvestre, An extension problem related to the fractional Laplacian, Comm. Partial Differential Equations 32 (2007), 1245-1260.

[5] L. A. Caffarelli and P. R. Stinga, Fractional elliptic equations, Caccioppoli estimates and regularity, Ann. Inst. H. Poincaré Anal. Non Linéaire 33 (2016), 767-807.

[6] Ó. Ciaurri, T. A. Gillespie, L. Roncal, J. L. Torrea, and J. L. Varona, Harmonic analysis associated with a discrete Laplacian, J. Anal. Math. 132 (2017), 109-131.

[7] Ó. Ciaurri, C. Lizama, L. Roncal, and J. L. Varona, On a connection between the discrete fractional Laplacian and superdiffusion, Appl. Math. Lett. 49 (2015), 119-125.

[8] F. del Teso, Finite difference method for a fractional porous medium equation, Calcolo 51 (2014), 615638.

[9] J. E. Galé, P. J. Miana, and P. R. Stinga, Extension problem and fractional operators: semigroups and wave equations, J. Evol. Equ. 13 (2013), 343-368.

[10] R. K. Getoor, First passage times for symmetric stable processes in space, Trans. Amer. Math. Soc. 101 (1961), 75-90.

[11] F. A. Grünbaum and P. Iliev, Heat kernel expansions on the integers, Math. Phys. Anal. Geom. 5 (2002), no. 2, 183-200.

[12] Y. Huang and A. Oberman, Numerical methods for the fractional Laplacian: a finite difference-quadrature approach, SIAM J. Numer. Anal. 52 (2014), 3056-3084.

[13] K. Kirkpatrick, E. Lenzmann, and G. Staffilani, On the continuum limit for discrete NLS with long-range lattice interactions, Comm. Math. Phys. 317 (2013), 563-591.

[14] N. S. Landkof, Foundations of Modern Potential Theory (Translated from the Russian by A. P. Doohovskoy), Die Grundlehren der mathematischen Wissenschaften 180, Springer-Verlag, New York, 1972.

[15] N. N. Lebedev, Special Functions and Its Applications, Dover, New York, 1972.

[16] M. Matsuki and T. Ushijima, A note on the fractional powers of operators approximating a positive definite selfadjoint operator, J. Fac. Sci. Univ. Tokyo Sect. IA Math. 40 (1993), 517-528.

[17] R. Metzler and J. Klafter, The random walk's guide to anomalous diffusion: a fractional dynamics approach, Phys. Rep. 339 (2000), 1-77.

[18] R. H. Nochetto, E. Otárola, and A. J. Salgado, A PDE approach to fractional diffusion in general domains: a priori error analysis, Found. Comput. Math. 15 (2015), 733-791.

[19] F. W. J. Olver and L. C. Maximon, Bessel Functions, NIST Handbook of Mathematical Functions (edited by F. W. J. Olver, D. W. Lozier, R. F. Boisvert and C. W. Clark), Chapter 10, National Institute of Standards and Technology, Washington, DC, and Cambridge University Press, Cambridge, 2010. Available online in http://dlmf.nist.gov/10

[20] A. P. Prudnikov, A. Y. Brychkov, and O. I. Marichev, Integrals and Series. Vol. 2. Special Functions, Gordon and Breach Science Publishers, New York, 1990.

[21] L. Roncal and P. R. Stinga, Transference of fractional Laplacian regularity, Special Functions, Partial Differential Equations and Harmonic Analysis. In honor of Calixto P. Calderón (edited by A. M. Stokolos, C. Georgakis and W. Urbina), pp. 203-212, Springer Proceedings in Mathematics and Statistics 108, Springer, 2014.

[22] L. Roncal and P. R. Stinga, Fractional Laplacian on the torus, Commun. Contemp. Math. 18 (2016), 1550033, 26 pp.

[23] O. Savin and E. Valdinoci, Density estimates for a nonlocal variational model via the Sobolev inequality, SIAM J. Math. Anal 43 (2011), 2675-2687.

[24] O. Savin and E. Valdinoci, Density estimates for a variational model driven by the Gagliardo norm, $J$. Math. Pures Appl. 101 (2014), 1-26.

[25] L. Silvestre, Regularity of the obstacle problem for a fractional power of the Laplace operator, Comm. Pure Appl. Math. 60 (2007), 67-112. 
[26] E. M. Stein, Singular Integrals and Differentiability Properties of Functions, Princeton Univ. Press, Princeton, NY, 1970.

[27] E. M. Stein and S. Wainger, Discrete analogues in harmonic analysis II: fractional integration, J. Anal. Math. 80 (2000), 335-355.

[28] P. R. Stinga, Fractional powers of second order partial differential operators: extension problem and regularity theory, PhD Thesis, Universidad Autónoma de Madrid, 2010.

[29] P. R. Stinga and J. L. Torrea, Extension problem and Harnack's inequality for some fractional operators, Comm. Partial Differential Equations 35 (2010), 2092-2122.

[30] F. G. Tricomi and A. Erdélyi, The asymptotic expansion of a ratio of Gamma functions, Pacific J. Math. 1 (1951), 133-142.

[31] A. Zoia, A. Rosso, and M. Kardar, Fractional Laplacian in bounded domains, Phys. Rev. E 76 (2007), 021116.

(Ó. Ciaurri and J. L. Varona) Departamento de Matemáticas y Computación, Universidad de La Rioja, 26006 Logroño, Spain

E-mail address: \{oscar.ciaurri,jvarona\}@unirioja.es

(L. Roncal) Basque Center for Applied Mathematics (BCAM), Alameda de Mazarredo 14, 48009 Bilbao, Spain

E-mail address: Ironcal@bcamath.org

(P. R. Stinga) Department of Mathematics, Iowa State University, 396 Carver Hall, Ames, IA, 50011, USA

E-mail address: stinga@iastate.edu

(J. L. Torrea) Departamento de Matemáticas, Facultad de Ciencias, Universidad Autónoma de MADRID, 28049 MADRID, SPAIN

E-mail address: joseluis.torrea@uam.es 